\title{
Unterricht aus der Sicht von Lehrenden und Lernenden
}

\author{
Passungen und Divergenzen zwischen Perspektiven \\ auf Klassengespräche
}

\begin{abstract}
Dieses Kapitel stellt zwei methodische Zugänge vor, mit deren Hilfe sequenzielle Analysen von (Unterrichts-)Interaktion angereichert werden können: Sie machen Wissensbestände sichtbar, die in der Interaktionsanalyse im Allgemeinen nicht rekonstruiert werden können, die aber für die Veränderung von Handlungsroutinen zentral sein dürften. Datengrundlage sind vier Gruppendiskussionen von Lehrpersonen sowie sechs von Schülerinnen und Schülern, in denen sich die Beteiligten handlungsentlastet jeweils über dieselben kurzen Videosequenzen aus dem Deutsch- und Mathematikunterricht von fünften Jahrgangsstufen verständigen. An diesen Interaktionen zweiter Ordnung wird einerseits untersucht, welche (sprachlichen) Aspekte von unterrichtlichen Diskursaktivitäten explizit thematisiert werden. Anderseits werden die Referenzformen auf Schülerinnen und Schüler als soziale Kategorisierungen betrachtet, die Hinweise geben auf die impliziten Orientierungen bezüglich der sozialen Ordnung des Klassenverbandes. Beide Zugänge ergeben im Vergleich jeweils innerhalb der Gruppen der Lehrenden und Lernenden sowie zwischen Lehrer- und Schülersicht Übereinstimmungen und Divergenzen, die bedeutsam sind für unser Verständnis von Passungen zu den institutionellen Zwecken und Erwartungen von Unterricht.
\end{abstract}

Keywords: Gruppendiskussion, Unterrichtsgespräche, Deutungsmuster, Kategorisierungen, membership categorization, Relevantsetzungen, soziale Ordnung

Vivien Heller, Bergische Universität Wuppertal, Gaußstraße 20, 42119 Wuppertal, vheller@uni-wuppertal.de

Uta Quasthoff, TU Dortmund, Emil-Figge-Straße 50, 44227 Dortmund, uta.quasthoff@tu-dortmund.de

Ә Open Access. ( 2021 Vivien Heller, Uta Quasthoff, publiziert von De Gruyter. (c))BY-NC-ND Dieses Werk ist lizensiert unter einer Creative Commons Namensnennung - Nicht-kommerziell - Keine Bearbeitung 4.0 International Lizenz.

https://doi.org/10.1515/9783110707168-014 


\section{Einführung: Unterschiedliche Perspektiven auf dieselben Unterrichtsprozesse und ihr analytisches Potenzial}

Betrachten wir die folgende Sequenz aus einem Deutschunterricht zu Beginn einer fünften Jahrgangsstufe, in der es um die Einrichtung eines häuslichen Arbeitsplatzes für die Schülerinnen und Schüler geht. In diesem Zusammenhang ist der Begriff ,Lexikon` gefallen:

Beispiel $^{1}$ (1a) 01-2-DU-1-120903-02-Lexikon (tru: Schülerin Trude, leh: Lehrer)

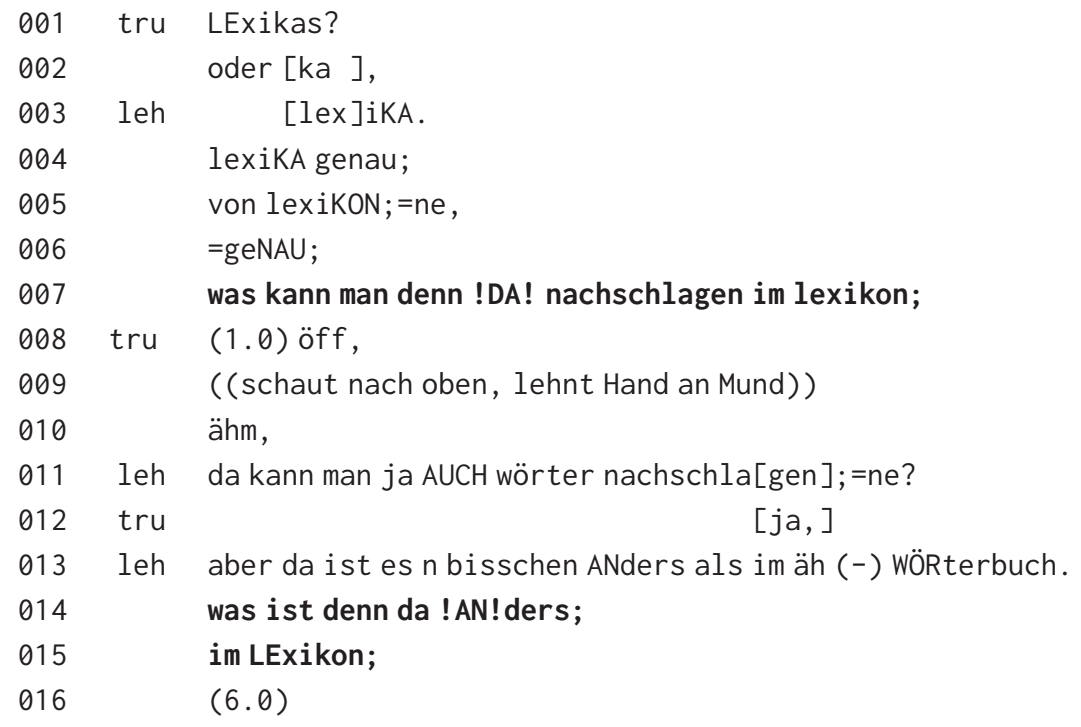

In erster Draufsicht handelt es sich hier um eine typische Sequenz eines Klassengesprächs zur Begriffsklärung, wesentlich gesteuert durch Fragen der Lehrperson, die von den Schülerinnen und Schülern nach Rederechterteilung bearbeitet werden. In einem etwas genaueren rekonstruktiv-analytischen Zugriff lassen sich bezogen bereits auf diesen kleinen Ausschnitt weitergehende Aussagen zu einzelnen Aspekten machen. Man kann bspw. - wie in der zugrundeliegenden Studie Inter-

1 Die Transkripte folgen der GAT 2 Notation (Selting et al. 2009; s. auch Anhang zu diesem Band). 
Pass $^{2}$ - die Art der lehrerseitigen Zugzwänge (im Transkript fett) deskriptiv betrachten sowie die Art, in der die Lehrperson sprachlich mit den anschließenden Äußerungen der Schülerinnen und Schüler umgeht (Buttlar 2019; Leßmann in diesem Band; Heller 2012; Heller \& Morek 2015; Margutti \& Drew 2014). Man kann mit Fokus auf die Lernenden und ihre Lernchancen fragen, welche Anschlussmöglichkeiten die Schülerinnen und Schüler jeweils auf die Zugzwänge der Lehrperson haben und welche davon sie umsetzen (Heller 2017; Koole \& Elbers 2014; Michaels \& Connor 2015; Morek 2016).

Welche analytischen Themen jeweils im Einzelnen fokussiert werden, hängt auch im Rahmen konversationsanalytisch basierter Verfahren von der Forschungsfrage ab, wodurch auch in dieser strikt an den Relevanzen der Beteiligten orientierten Vorgehensweise der Blickwinkel der Forschenden ins Spiel kommt. Genau betrachtet und analytisch rekonstruiert wird an den aufgezeichneten Sequenzen unter der Vielzahl des Beobachtbaren immer nur das, was für deren Beantwortung relevant ist. Da sich ein konversationsanalytischer Forschungsprozess bekanntermaßen ausschließlich an dem zu orientieren hat, was für die Beteiligten im Vollzug der Interaktion audible and visible ist (Schegloff 1991), lassen sich nicht alle Forschungsfragen auf der Grundlage rein sequenzieller Daten bearbeiten. Fundierte Aussagen im Rahmen des breiteren Erkenntnisinteresses von InterPass zu Partizipationschancen oder Lerngelegenheiten, die die betrachtete Unterrichtsform den Schülerinnen und Schülern jeweils bietet (vgl. Quasthoff, Heller \& Morek in diesem Band), verlangen z. B. bereits eine breitere Datenbasis, etwa in Form einer längsschnittlichen Beobachtung einzelner Schülerinnen und Schüler (Cekaite 2012; Heller 2017; Quasthoff \& Prediger 2017; van der Veen, Mey et al. 2017).

Spätestens sobald die jeweilige Fragestellung Anwendungsaspekte einschließt, die über die Rekonstruktion vorfindlichen Verhaltens hinaus langfristig auf die Veränderung einer rekonstruierten Praxis seitens der institutionell Handelnden abzielen, braucht man aus unserer Sicht zusätzlich Erkenntnisse, die nicht (nur) mit Hilfe sequenzieller Analysen beobachtbarer Praktiken zu erhalten sind. Betrachten wir zur besseren Fundierung des Gedankengangs die Fortsetzung des in Beispiel (1a) dokumentierten Unterrichtsgeschehens:

2 „Interaktive Verfahren der Etablierung von Passungen und Divergenzen für sprachliche und fachkulturelle Praktiken im Deutsch- und Mathematikunterricht. Eine rekonstruktive Unterrichtsstudie zur Teilhabe an schulischen Vermittlungsprozessen“, gefördert mit Mitteln des Bundesministeriums für Bildung und Forschung (Förderkennzeichen 01JC1112; Projektleitung S. Prediger \& U. Quasthoff). 
Beispiel (1b)

\begin{tabular}{|c|c|c|}
\hline 017 & leh & FEli, \\
\hline 018 & fel & dass man zum beispiel einen BUCHstaben hat, \\
\hline 019 & & zum beispiel KA:, (.) \\
\hline 020 & & und dann ist das äh einmal KlEIN, = \\
\hline 021 & & =und einmal GROß geschrieben,= \\
\hline 022 & & =und da steht zum beispiel DIE katze daneben, \\
\hline 023 & & und die ka (.) also katze (.) daneben so, \\
\hline 024 & leh & {$\left[\mathrm{hm} \_\mathrm{hm}, \mathrm{]}\right.$} \\
\hline 025 & fel & [und DIE] katze steht daneben als ähm (. ) verb; \\
\hline 026 & & $(4.0)$ \\
\hline 027 & & $<<p>$ so weit WAR ich $>$. \\
\hline 028 & leh & ((legt Kopf schief, runzelt Stirn)) \\
\hline 029 & & $<<$ in die Klasse blickend $>$ WIE ist es im lexikon, = \\
\hline 030 & & =und WIE ist es im wörterbuch>. \\
\hline 031 & fel & |ach SO: ; \\
\hline & & $\mid(($ lässt Kopf nach vorne fallen $)) \mid$ \\
\hline 032 & & $(3.0)$ \\
\hline
\end{tabular}

Eine sequenzielle Analyse zeigt: Feli liefert einen Erklärversuch (Z. 18-23), der von der Lehrperson nur „minimal ratifiziert“ (Buttlar 2019; Leßmann i. E.) wird (Z. 24), und schließlich durch eine Wiederholung des Zugzwangs, diesmal an die gesamte Klasse gerichtet (Z. 29/30), übergangen wird. Dies geschieht ohne Hinweis darauf, warum der Beitrag aus Sicht der Lehrperson nicht passt. Handlungsleitende professionelle Überzeugungen und Orientierungen der Lehrperson (Baumert \& Kunter 2006; Reusser \& Pauli 2014), die sie zu ihren Zügen in der Unterrichtsinteraktion veranlassen (hier: das Übergehen von Felis Beitrag in Z. 29/ 30) und die mit dem Ziel einer Veränderung des lehrerseitigen Verhaltens ggfs. bearbeitet werden müssten, bleiben einer rein rekonstruktiv vorgehenden Analyse auch in dem längeren Ausschnitt aus dem Klassengespräch jedoch verborgen. Auch Felis innere Reaktion auf die implizite Ablehnung ihrer Antwort seitens der Lehrperson ist nicht zugänglich.

Beides, professionelle Überzeugungen und Orientierungen auf Lehrerseite ebenso wie schülerseitiges Erleben und Bewerten, ist jedoch für einen anwendungsbezogenen erklärenden Zugang zur rekonstruierten Praxis entscheidend: Auf Grundlage welcher Überzeugungen und Orientierungen agieren Lehrpersonen in den Interaktionsmustern, die die sequenzielle Bearbeitung aufdeckt? Wie erleben unterschiedliche Schülerinnen und Schüler diese Musterhaftigkei- 
ten, bspw. die ausbleibende explizite Rückmeldung oder zweite Chance, in ihrem Schulalltag?

Ohne einen empirischen Zugang zu diesem (schul-)alltagsweltlichen Wissen, das in seiner Selbstverständlichkeit im Handlungsvollzug typischerweise nicht expliziert wird und entsprechend sequenziell nicht rekonstruierbar ist, können Motive und Wirkungen des lehrerseitigen Interaktionshandelns nicht hinreichend erfasst und damit das Handeln selbst über die sequenzielle Mechanik hinaus nicht verstehbar werden. Eine tiefere Einbettung der rekonstruierbaren Musterhaftigkeiten durch ethnographische Zugänge reichert das Forschungsfeld also in einer Weise an, die später wesentlich ist im Versuch, Lernchancen vorfindlicher Unterrichtspraktiken einzuschätzen und professionelle Routinen nachhaltig zu verändern. Die impliziten Überzeugungen und Orientierungen der Lehrkräfte sind dabei u. a. deshalb zu berücksichtigen (Prediger in diesem Band), weil sie ihr Routinehandeln auch in solchen Bereichen erklären können, die ihnen selbst nicht immer bewusst zugänglich sind. Ebenso sollte der Einblick in die verborgenen Deutungen und Bewertungen auf Schülerseite einen wichtigen Baustein zur Einschätzung der Lernchancen liefern, weil bspw. die Erfahrung von Wertschätzung und Akzeptanz seitens der Schülerinnen und Schüler eine zentrale Rolle für den Lernerfolg spielt. Es wird also darauf ankommen, einen Zugang gerade auch zu impliziten, in der Interaktion nicht selbst zum Thema gemachten Alltagskonzepten der sozialen Ordnung im Klassenzimmer zu finden. Der vorliegende Beitrag präsentiert methodische Vorschläge zur Umsetzung dieser Forderung auf der Grundlage von Gruppendiskussionen zu Unterrichtssequenzen.

Betrachten wir zur Konkretisierung des Gesagten und zur Demonstration des analytischen Potenzials unseres recht ungewöhnlichen empirischen Zugangs einführend zunächst an einigen Beispielen, in welcher Weise die Dokumentation des Unterrichtsgesprächs (1a und 1b) selbst angereichert wird durch Aussagen, die andere Lehrende und Lernende in Gruppendiskussionen - also handlungsentlastet - dazu gemacht haben. Die Diskutierenden wurden dabei mit videographierten und transkribierten Unterrichtssequenzen konfrontiert (zur genauen Beschreibung der Gruppendiskussionsdaten s. Abschnitt 3).

Ein Mathematiklehrer aus einer Diskussion unter Gymnasiallehrkräften kommentiert z. B. typisches Lehrerhandeln und bemerkt zum „Nachschieben von Fragen“ (in Beispiel 1a: Z. 7, 14-15):

Beispiel (2) L-GD 1 (Gym, ML+DL)

1874 nes ist ja so_n TYpisches phänomen.

1875 anstatt einfach mal ABzuwarten. 
Ein Schüler findet:

Beispiel (3) Sch-GD 1 (Jungen Gym, L1/L2)

1111 ole aber der LEHrer ist jetzt auch ganz schön nett-

Die beiden folgenden Ausschnitte von Deutschlehrerinnen thematisieren auf unterschiedliche Weise heterogene Lernvoraussetzungen:

Beispiel (4) L-GD 4 GS + Gym DL

1401 böl also ich ich spreche da aus erFAHrung?

1402 es gibt manchmal (.) wÖRter die tauchen auf,

1403 von denen (. ) geht man nicht AUS dass die_n problem darstellen?

1404 also gerade bei meinem klienTEL (. ) ähm.

1405 sowas wie SEUFzen oder flüstern?

1406 und dann FRAgen die,

1407 was ist denn FLÜstern.

1408 ähm (. ) da muss man SCHON mal kurz drüber nachdenken.

Beispiel (5) L-GD 4 GS + Gym DL

1590 pil ja wenn er wenn du inner diskusSION steckst mit höheren scHülern oder?

1591 dann denkste naja gut jetzt PASST das oder nicht.

1592 aber eben in der FüNFten klasse;

1593 wenn ich jetzt so bespreche was jetzt ein LExikon ist oder ein duden oder ein wörterbuch, es ist vom SPRACHverhalten schon (--) n bisschen höher. also mei manche in MEIner klasse würden mit diesem sachverhalt im grunde erstmal gucken$<<$ animiert> was ist !DAS! denn.

Wir können also bereits auf der Grundlage dieser wenigen Äußerungen zu einem kurzen Videoclip festhalten, dass Diskussionsbeteiligte sehr unterschiedliche Aspekte desselben gezeigten Videoausschnitts thematisieren: Gesamtschullehrkräfte thematisieren z. B. - im Unterschied zu gymnasialen Lehrkräften (s. u. Abschnitt 5.2) - die unterschiedlichen lese- und sprachbezogenen häuslichen Vorerfahrungen der Fünftklässler. Die Schülerinnen und Schüler wiederum formulieren personenbezogene Einschätzungen der handelnden Lehrpersonen wie „nett“. 
Aus sprachdidaktischer Sicht ergibt sich im Zusammenhang mit dem analytischen Potential dieses Zugangs die Frage, in welchem Ausmaß die Sprachlichkeit des Unterrichtsgeschehens und damit die Rolle sprachlicher Kompetenzen von Schülerinnen und Schülern dabei überhaupt in den Blick von Lehrenden und Lernenden gerät (s. bspw. Beispiel 4 und 5). In Abschnitt 4 beschäftigen wir uns deshalb mit der Frage, was Diskussionsbeteiligte im Rahmen der Betrachtung von Schülerbeiträgen insgesamt thematisieren. Dabei fokussieren wir auf die diskursiven Prozesse des Erklärens und Begründens, die aus der Erwerbsperspektive im Sprachlernkontext Unterricht einen zentralen Stellenwert einnehmen (Heller, Quasthoff, Prediger et al. 2017; Muller Mirza \& Perret-Clermont (Hrsg.) 2009; Resnick, Asterhan \& Clarke 2015; Redder 2016). Wir fragen in diesem Zusammenhang, welche Aspekte von Schüleräußerungen in den Diskussionsbeiträgen im Wortsinne thematisch relevant gesetzt werden.

Neben diesen Beobachtungen zur Unterschiedlichkeit der thematisierten Relevantsetzungen von Schüleräußerungen präsentieren wir einen weiteren analytischen Zugang zu den Gruppendiskussionsdaten, der auf implizite Alltagswissensbestände zur sozialen Ordnung und zu sozialen Kategorisierungen der Beteiligten im Klassenzimmer abzielt. Hierzu nutzen wir die kategorialen Bezeichnungen, mit denen Sprechende Referenz auf Personen herstellen: Oft ist z. B. von „Kindern“, dann wiederum von „Schülern“ die Rede, in Beispiel (4) von „meinem Klientel“ und in Beispiel (5) werden „höhere Schüler“ mit „fünften Klassen“ kontrastiert. Alle Referenzformen mit Ausnahme der „höheren Schüler“ beziehen sich im Kontext des Videos abstrakt oder konkret in jeweils unterschiedlicher semantischer Perspektivierung auf dieselben Referenten, Schülerinnen und Schüler in der fünften Jahrgangsstufe. Wir werden im Abschnitt 5 entfalten, welchen Einblick derartige nominale Referenzformen in implizite Deutungen der sozialen Ordnung des Klassenzimmers gewähren.

Alle beispielhaft gezeigten Deutungen aus den Gruppendiskussionen wären so nicht aus den Unterrichtssequenzen selbst rekonstruierbar gewesen, spielen aber - so ist zu unterstellen - im habituellen und andauernden Vollzug des Unterrichts für die Beteiligten eine Rolle. Unter dem eingangs thematisierten Aspekt der Veränderbarkeit von Lehrerhandeln führt der nachfolgende Beitrag also zwei grundlegende analytische Zugänge zu Konfrontationsdaten vor: (1) Thematische Relevantsetzungen legen offen, was die Diskussionsbeteiligten an den jeweiligen Beiträgen für wesentlich halten und welche Rolle sprachliche Aspekte dabei spielen. Diese Ergebnisse geben darüber Aufschluss, ob Partizipationsgelegenheiten und die damit verbundenen sprachlich-diskursiven Anforderungen der Unterrichtsgespräche einschließlich der möglichen schülerseitigen Schwierigkeiten bzw. Unterstützungsbedarfe bisher überhaupt in den Blick von Lehrenden 
und Lernenden geraten. (2) Implizite Hinweise auf soziale Kategorisierungen legen offen, wie die soziale Ordnung der Agierenden im institutionellen Unterrichtsgeschehen jeweils perspektiviert wird. Systematische Diskrepanzen zwischen Lehrenden und Lernenden geben z. B. Aufschluss darüber, welche wechselseitigen Erwartungen verändert werden müssten, damit Passungen zwischen lehrerseitigen Partizipationsbemühungen und schülerseitiger Partizipationsbereitschaft überhaupt denkbar werden.

Bevor diese analytischen Komplexe in den Abschnitten 4 und 5 bearbeitet werden, verorten wir im folgenden Abschnitt unseren Ansatz im Kontext relevanter Forschungen $\mathrm{zu}$ Sichtweisen von Lernenden und Lehrenden auf Unterricht und beschreiben im Abschnitt 3 unsere Daten sowie die konkreten methodischen Vorgehensweisen.

\section{Forschungen zu Sichtweisen von Lernenden und Lehrenden auf Unterricht}

Untersuchungen zu Sichtweisen von Lehrenden und Lernenden auf Unterricht sind vordringlich in der bildungswissenschaftlichen Unterrichtsqualitätsforschung verortet. Pädagogisch-psychologische Untersuchungen, die nicht der Unterrichtsqualität gewidmet sind, analysieren Typisierungen, die sich in den Wahrnehmungen der Lernenden durch die Lehrkräfte manifestieren. Soziologisch und gesprächsanalytisch orientierte Arbeiten fokussieren auf Prozesse der Kategorisierung in situ und rekonstruieren gesellschaftlich sedimentierte und geteilte soziale Kategorien; in diesem Feld liegen u. a. Arbeiten zu Typisierungen und sozialen Kategorisierungen im Rahmen von schulischen Sprechstunden oder Lehrerkonferenzen vor.

Wenngleich es im Rahmen der Unterrichtsqualitätsforschung schwerpunktmäßig um die professionelle Unterrichtswahrnehmung durch Lehrende geht, da diese eine zentrale Voraussetzung für eine hohe Unterrichtsqualität bildet, wird auch die Sicht von Lernenden auf Unterricht als eine Perspektive eigenen Rechts in den Blick genommen. Bei der Erfassung der schüler- und lehrerseitigen Wahrnehmung kommen i.d.R. standardisierte Befragungsinstrumente (Ditton 2002), z. T. in Verbindung mit Videoaufzeichnungen des eigenen oder auch fremden Unterrichts zum Einsatz (z. B. Baumert et al. 2004; Seidel \& Prenzel 2008; Holodynski et al. 2017), um professionsbezogene Wissensstrukturen bezüglich lernwirksamer Unterrichtssituationen situativ, authentisch und kontextbezogen zu erfassen.

Ditton (2002) befragt in 186 neunten Klassen Lernende zur Wahrnehmung ihres Mathematikunterrichts und der Mathematik-Lehrkraft sowie die entspre- 
chenden Lehrpersonen zur Wahrnehmung durch die jeweilige Schulklasse. Die Mehrebenenanalysen zeigen, dass die Wahrnehmung der Lehrkräfte durch die Schülerinnen und Schüler primär mit der Wahrnehmung der Unterrichtsqualität zusammenhängt. Lernende beurteilen Mathematiklehrkräfte dann als positiv, wenn sich letztere durch einen klar strukturierten Unterricht, eine hohe diagnostische Kompetenz und ein gutes Verhältnis zu den Lernenden auszeichnen. Die Befragung der Lehrpersonen offenbart, dass diese ihre Wirkung auf die Schülerinnen und Schüler überwiegend gut einschätzen können; bei einem Teil der Lehrkräfte zeigen sich allerdings Divergenzen zu den Wahrnehmungen der Lernenden. Hinsichtlich seiner methodischen Herangehensweise hält Ditton einschränkend fest, dass die wechselseitige Wahrnehmung von Lernenden und Lehrenden im Unterricht auch mit elaborierten quantitativen Erhebungsund Analyseverfahren nur begrenzt abgebildet werden kann.

Im Unterschied zu Ditton zeigen sich in Claussens (2002) Untersuchung zur Wahrnehmung der Qualität des Mathematikunterrichts durchgängige Divergenzen zwischen den Wahrnehmungen von Lernenden und Lehrenden. Die Datengrundlage bilden Fragebogendaten von Lernenden und Lehrkräften aus 53 Längsschnittklassen der TIMSS-Studie, die mit Einschätzungen externer Videobeurteiler verglichen wurden. Der Grad der Übereinstimmung hängt Claussen zufolge von dem jeweiligen Unterrichtsmerkmal, genauer: von dessen Beobachtbarkeit, ab. Ebenfalls mit einem Fokus auf den Mathematikunterricht untersuchen Baumert et al. (2004) in einer Zusatzstudie zu PISA 2003 die Wahrnehmung von Merkmalen der Unterrichtsqualität bei Lernenden und Lehrenden. Letztere werden jeweils zu dem von ihnen erteilten bzw. erlebten Unterricht befragt. Bei den Lehrpersonen zeigen sich schulformspezifische Unterschiede in der Wahrnehmung des eigenen Unterrichts: Gymnasiallehrkräfte nehmen ihren Unterricht tendenziell als kognitiv-aktivierend wahr, während Lehrkräfte von Schulen mit mehreren Bildungsgängen ihren Unterricht als enggeführt einschätzen. Aus Schülersicht ist unter mehreren thematisierten Merkmalen insbesondere das professionelle Ethos der Lehrperson entscheidend für die Unterrichtsqualität. Auch hier zeigen sich schulformspezifische Unterschiede: Hauptschülerinnen und -schüler beurteilen bspw. die Angebotsstruktur des Mathematikunterrichts als positiv, während Gymnasiasten ihren Unterricht eher kritisch bewerten. Diese Differenzen führen die Autoren vor allem auf Unterschiede in der individuellen Lernunterstützung durch die Lehrkräfte zurück, welche von Hauptschülerinnen und -schülern als besonders ausgeprägt, von Gymnasialschülerinnen und -schülern hingegen als gering beschrieben wird. Mit Blick auf die Korrelation zwischen den Wahrnehmungen der Lehrenden und der Lernenden werden insbesondere in solchen Klassen Divergenzen sichtbar, die basierend auf den Lehrerangaben als problematisch eingestuft wurden. 
Im Rahmen der DESI-Studie setzen Klieme et al. (2008) Ergebnisse der Befragung von Lehrenden und Lernenden zu Merkmalen der Qualität des Deutschunterrichts mit der schülerseitigen Kompetenzentwicklung in Beziehung. Es zeigen sich Zusammenhänge zwischen der Leistungsentwicklung und dem wahrgenommenen Tempodruck, Anforderungsniveau sowie positiv wahrgenommener Disziplin.

Die genannten Studien machen deutlich, dass Unterricht von Lehrenden und Lernenden in unterschiedlicher Weise wahrgenommen werden kann. Aufgrund der vorstrukturierten und standardisierten Befragungsinstrumente wird die Einschätzung der Befragten allerdings stark gesteuert. Die Studien können dementsprechend keinen Aufschluss darüber geben, welche Aspekte von Unterricht Lehrende und Lernende ohne eine solche Lenkung thematisieren.

In dieser Hinsicht interessante Befunde liefert die pädagogisch-psychologische Studie von Hofer (1981), die untersucht, wie Deutschlehrkräfte Schülerinnen und Schüler wahrnehmen bzw. kategorisieren. Dazu werden 15 Lehrkräfte gebeten, ihre Schülerinnen und Schüler hinsichtlich vorgegebener Eigenschaftsskalen zu beurteilen. Über Jahrgangsstufen hinweg zeigt sich, dass Lehrpersonen Schülerinnen und Schüler nicht als Individuen wahrnehmen, sondern diese u. a. nach einer überschaubaren Anzahl von Kategorien (u. a. Begabung, Anstrengung, Diszipliniertheit, soziale Aktivität) typisieren. Hofer nimmt an, dass solche Typisierungen notwendig sind, um im Unterrichtsprozess Handlungsentscheidungen treffen zu können. Diesbezüglich lässt sich auch nachweisen, dass die vorgenommenen Typisierungen mit dem Handeln - bspw. der Häufigkeit von Fragekontakten und dem Rückmeldeverhalten - der Lehrpersonen zusammenhängen: So werden etwa als undiszipliniert und wenig begabt wahrgenommene Schülerinnen und Schüler im Vergleich häufiger getadelt und weniger gefördert. Dies kann Ausdruck des Bemühens sein, so Hofer, den Unterricht im Fluss zu halten.

Im Unterschied zu den genannten Studien zielen gesprächsanalytische Untersuchungen - seien sie soziologischer oder linguistischer Provenienz - nicht darauf ab, individuelle wahrnehmungsstrukturierende oder handlungsleitende Kognitionen $\mathrm{zu}$ erfassen, sondern sozial geteilte Kategorien und Kategorisierungsverfahren als Ressourcen der Gesprächsbeteiligten zu untersuchen. So zeigen Mazeland \& Berenst (2008), dass Lehrpersonen in Zeugniskonferenzen zunächst accounts und andere Formen von Belegen liefern, um dann einzelne Schülerinnen und Schüler institutionell relevanten Typen zuordnen. Diese Art der Kategorisierung resultiert in kollektiv geteilten Sichtweisen (Cedersund \& Svendsson 1996) und hat Konsequenzen für die Schullaufbahn (Mazeland \& Berenst 2008). Kotthoff (2012, 2014) rekonstruiert Kategorisierungen („faul wie e Hund“) im Rahmen von schulischen Sprechstunden, d.h. Gesprächen von Lehrkräften und Eltern über deren Kinder. Kategorisierungen erfolgen u. a. durch positive/negative Bewertungen, Etikettierungen („Luftikus“, vgl. Kotthoff 
2012: 315) und Prädikationen (,ist nicht bei der Sache“, Kotthoff 2012: 315). Sie werden nicht nur von den Lehrpersonen, sondern auch von den Eltern produziert, die sich auf diese Weise im Gespräch mit der Lehrperson als schulkompetent und die Maßnahmen der Lehrperson unterstützend positionieren.

Eine solche rekonstruktive Herangehensweise greifen wir im Folgenden auf, indem wir Gruppendiskussionen von Lehrpersonen und Schülerinnen und Schülern über dieselben Unterrichtssequenzen mit Blick auf thematische Relevantsetzungen und implizite Kategorisierungen untersuchen und die jeweiligen Sichtweisen in Beziehung setzen.

\section{Daten und Vorgehensweise}

Die im vorliegenden Beitrag genutzten Daten wurden als Teilstudie von InterPass (Quasthoff \& Prediger 2017) im Rahmen der in Abschnitt 1 skizzierten Fragestellungen erhoben und mit der Unterrichtsstudie, die Unterrichtsinteraktionen mikroanalytisch untersuchte, trianguliert. Die Perspektiven von Lehrkräften auf Unterrichtsinteraktionen wurden dort mit Hilfe von vier Gruppendiskussionen (die ihrerseits videographiert wurden) von jeweils 95-110 Minuten Dauer erhoben. Die einzelnen Diskussionsgruppen der Lehrpersonen bestanden aus je 5-9 Lehrkräften. $\mathrm{Zu}$ den Lernenden wurden sechs Diskussionsgruppen aufgezeichnet, die aus je 5-6 Fünftklässlerinnen und Fünftklässlern bestanden. Sie dauerten jeweils etwa 90 Minuten. Alle Diskussionsgruppen wurden mit denselben kurzen Videoclips aus Deutsch- und Mathematikstunden konfrontiert. Zur Wahrung der Anonymität der im Dortmunder Raum videographierten Unterrichtsbeteiligten wurden die Gruppendiskussionen in Frankfurt und Hannover durchgeführt.

Die Zusammensetzung der Gruppen sollte dabei sicherstellen, dass die handlungsentlastet Diskutierenden jeweils etwa den gleichen Member-Status haben wie die unterrichtlich Handelnden, damit wir im Rahmen der Gruppendiskussionsmethode (Bohnsack 2010; Przyborski 2004; Wagner-Willi 2010) das im jeweiligen Handlungsfeld einschlägige kollektive Alltagswissen erfassen können. Wir haben in diesem Zusammenhang darauf verzichtet, die unterrichtlich handelnden Lehrenden und Lernenden selbst mit ihrem eigenen aufgezeichneten Handeln zu konfrontieren (Auer 1995), u. a. weil wir keinen Legitimationsdiskurs initiieren und erfassen wollten.

Die vier Gruppendiskussionen mit Lehrkräften setzen sich entsprechend zusammen aus Beteiligten mit Erfahrungen, die denen in der Unterrichtsstu- 
die in Klasse 5 videographierten Lehrkräfte vergleichbar sind, und zwar mit Blick auf

- die Schulart,

- die unterrichteten Fächer,

- die Erfahrungen mit fünften Klassen und

- den sozialen und sprachlichen Hintergrund der Schülerinnen und Schüler.

Beide Schulformen (Gymnasial- und Gesamtschullehrkräfte) und Fachhintergründe (Deutsch und Mathematik) wurden abgedeckt und in den Gruppenzusammensetzungen variiert (s. Tabelle 1):

Tab. 1: Zusammensetzung der Lehrer-Gruppen.

\begin{tabular}{lllr}
\hline Gruppe & $\begin{array}{l}\text { Zusammensetzung } \\
\text { (Schulart, Fach) }\end{array}$ & Gruppengröße \\
\hline Gruppe 1 & Gymnasium & Mathe/Deutsch & 5 \\
Gruppe 2 & Gesamtschule & Mathe/Deutsch & 7 \\
Gruppe 3 & Ges/Gym & Mathe & 7 \\
Gruppe 4 & Ges/Gym & Deutsch & 9 \\
\hline
\end{tabular}

Insgesamt waren 28 Lehrkräfte beteiligt, davon 14 Lehrer und 14 Lehrerinnen.

Die sechs Gruppendiskussionen der Lernenden umfassten insgesamt 34 Kinder der Jahrgangsstufe 5 (10-11 Jahre), davon 23 Schüler und 11 Schülerinnen. 12 besuchten ein Gymnasium, 22 eine Gesamtschule. Bei der Zusammensetzung der Schüler-Diskussionsgruppen wurden Schulform, der sozioökonomische Status (SES; kategorisiert nach Einzugsbereich der Schule und der Zuschreibung durch die Lehrpersonen), die Sprachbiographie (Deutsch als Erst- bzw. Zweitsprache, operationalisiert nach Selbstauskunft zur Familiensprache) sowie das Geschlecht (mit einem leichten Übergewicht von Jungen) berücksichtigt. Tabelle 2 zeigt die Zusammensetzung der Schüler-Gruppen:

Tab. 2: Zusammensetzung der Schüler-Gruppen.

\begin{tabular}{|c|c|c|c|c|}
\hline \multirow{2}{*}{$\begin{array}{l}\text { Gruppe } \\
\text { Gruppe HJ1 }\end{array}$} & \multicolumn{3}{|c|}{$\begin{array}{l}\text { Zusammensetzung } \\
\text { (Schulart, SES, Ein-/Mehrsprachigkeit, Geschlecht) }\end{array}$} & \multirow{2}{*}{ Gruppengröße } \\
\hline & Gymnasium & high SES (L1 und L2) & Jungen & \\
\hline Gruppe HJ2 & Gymnasium & high SES (L2) & Jungen & 6 \\
\hline Gruppe LJ3 & Gesamtschule & low SES (L1 und L2) & Jungen & 6 \\
\hline Gruppe LJ4 & Gesamtschule & low SES (L2) & Jungen & 5 \\
\hline
\end{tabular}


Tab. 2 (fortgesetzt)

\begin{tabular}{llllr}
\hline Gruppe & \multicolumn{2}{l}{ Zusammensetzung } & & Gruppengröße \\
& (Schulart, SES, Ein-/Mehrsprachigkeit, Geschlecht) & \\
\hline Gruppe LM5 & Gesamtschule & low SES (L1 und L2) & Mädchen & 6 \\
Gruppe LM6 & Gesamtschule & low SES (L2) & Mädchen & 5 \\
\hline
\end{tabular}

Insgesamt wurden bei den Lehrer-Gruppendiskussionen 7 Stunden und 15 Minuten und bei den Schüler-Gruppendiskussionen 8 Stunden und 6 Minuten videographiert. Die videographierten Gruppendiskussionen wurden anschließend vollständig nach GAT 2 (Detaillierungsgrad: Minimaltranskription, vgl. Selting et al. 2009; zu den wichtigsten Notationen s. Anhang) transkribiert.

In der Durchführung wurden in allen Diskussionsgruppen etappenweise dieselben fünf kurzen Videosequenzen aus dem Datenkorpus der Interpass-Unterrichtsstudie von jeweils 1-4 Minuten Dauer präsentiert. Eine comicartig gestaltete Transkription gewährte dabei leichten Zugang zum Wortlaut der Äußerungen. In den Clips waren typische Unterrichtsinteraktionen zwischen Lehrperson und Schülerinnen und Schülern zu sehen, die sich $u$. a. durch eine höhere oder geringere Passung des Schüler-Beitrags zum Zugzwang der Lehrperson unterschieden. Die Clips enthielten Szenen aus Deutsch- und Mathematikstunden unter Beteiligung sowohl von Lernenden mit Deutsch als Herkunfts- wie als Zweitsprache; sie wurden in festgelegten Stopps nach jeweils kurzen Etappen präsentiert.

Über die einzelnen Videos und die vorbereiteten Stopps hinaus gaben die Forschenden nur wenige Impulse, um gemäß den Prinzipien der Gruppendiskussionsmethode nicht in die Dynamik des Meinungsprozesses in der Gruppe (Bohnsack 2010) und die thematischen Relevantsetzungen einzugreifen.

\section{Thematische Relevantsetzungen im Umgang mit Erklärungen und Begründungen}

Die Videokonfrontationen im Rahmen der Gruppendiskussionen für die vorliegende Studie haben den Stellenwert eines Interaktionsereignisses eigener Art. Sie liefern gleichsam Beobachtungen zweiter Ordnung (Luhmann 1997): Vom Handlungsdruck entlastete Lehrende und Lernende beobachten und kommentieren Unterricht. In einem ersten Schritt rekonstruieren wir nun, was sie in der Diskussion der beobachteten Interaktionen thematisch relevant setzen bzw. innerhalb welcher Orientierungsrahmen - bspw. Denk- und Sprachvermögen, Identität oder 
ethnische Herkunft - sie über Schüleräußerungen sprechen. In der Bearbeitung dieser Frage erhalten wir Aufschluss über kollektive Deutungsmuster (Meuser \& Sackmann 1992) von Lehrenden (unterschiedlicher Fächer und Schulformen) und Lernenden (unterschiedlicher soziokultureller Milieus). Mit Bezug auf die Wissenssoziologie Mannheims (1980) gehen wir davon aus, dass Lehrpersonen sowie Schülerinnen und Schüler jeweils eigene „konjunktive Erfahrungsräume“ (s. auch Wagner-Willi 2010) teilen. In diesen tradieren sich Deutungsmuster, d. h. überindividuell (re-)produzierte und zumeist präreflexive Interpretationsweisen wiederkehrender Ereignisse wie bspw. Lehrer-Schüler-Interaktionen. Eine interessante Frage ist nun, ob die soziale Veranstaltung Unterricht für Lehrende und Lernende jeweils einen anderen konjunktiven Erfahrungsraum darstellt. Darüber hinaus stellt sich die Frage, ob Schülerinnen und Schüler unterschiedlicher sozioökonomischer Milieus und sprachlicher Biografien in der kollektiven Deutung von Unterricht gruppenspezifische Muster aufweisen.

\subsection{Vorgehen in der Analyse}

Zunächst wurden in den Gruppendiskussionen diejenigen Sequenzen identifiziert, in denen sich die Beteiligten überhaupt auf die in den fünf Videoclips gezeigten Schüleräußerungen (und nicht etwa das didaktische Agieren der Lehrperson) bezogen. Bei den vier Gruppendiskussionen der Lehrerinnen und Lehrer waren dies 75 Sequenzen, bei den sechs Gruppendiskussionen der Schülerinnen und Schüler 213 Sequenzen. ${ }^{3}$ Diese Sequenzen bilden die Grundlage für die Analyse thematischer Relevantsetzungen.

Die mikroanalytische Beschreibung erfolgt sodann im Hinblick auf zwei Gesichtspunkte. Zum einen wird rekonstruiert, was thematisiert wird. Dazu wird in einem ersten Schritt in den Blick genommen, auf welchen Ausschnitt einer Sequenz referiert wird. Dies kann die gesamte Sequenz mit Lehrerfrage, Schülerantwort und lehrerseitiger Reaktion sein oder aber nur die Schüleräußerung bzw. einzelne Elemente. In einem zweiten Schritt wird untersucht, innerhalb welches thematischen Rahmens - bspw. der Form einer Äußerung, des Denkvermögens oder Wissens des Sprechenden usw. - der jeweilige Ausschnitt betrachtet wird.

Zum anderen wird sequenziell rekonstruiert, wie, d. h. mittels welcher sprachlichen Verfahren die Diskussionsbeteiligten bestimmte Aspekte hervorheben und

3 Für die unterschiedlichen Anzahlen sind zwei Gründe verantwortlich. Erstens war die Anzahl der Gruppendiskussionen der Lehrpersonen eine andere als die der Lernenden. Zweitens fokussierten die Lehrpersonen häufig das didaktische Agieren der beobachteten Lehrperson, so dass sich insgesamt weniger Bezugnahmen auf schülerseitige Erklärungen und Begründungen finden. 
thematisieren. Die rekonstruierten thematischen Relevantsetzungen reflektieren somit, wie und innerhalb welches thematischen Rahmens schülerseitige Erklärungen und Begründungen (vgl. zu diesen und anderen grundlegenden Konzepten das Glossar in diesem Band) musterhaft gedeutet werden. In einem weiteren Schritt wurden die Relevantsetzungen hinsichtlich ihrer Häufigkeit verglichen, um Aussagen zu gruppenübergreifenden Deutungen treffen zu können.

Die Analyse der lehrerseitigen thematischen Relevantsetzungen war bereits Gegenstand einer früheren Darstellung (Heller, Quasthoff, Prediger et al. 2017), weshalb auf sie hier nur vergleichend Bezug genommen wird. Die Analyse der schülerseitigen Relevantsetzungen förderte allerdings weitere Kategorien zutage. Aus diesem Grund erfolgt eine Reanalyse der Lehrer-Gruppendiskussionen, die in den Vergleich der Häufigkeiten thematischer Relevantsetzungen (Abschnitt 4.4) einfließt.

Im Folgenden werden die thematischen Relevantsetzungen mikrostrukturell beschrieben (4.2). Im Anschluss wird die Frequenz thematischer Relevantsetzungen zunächst mit Blick auf unterschiedliche Schülergruppen (4.3) und anschließend mit Blick auf Unterschiede zwischen Lehrpersonen und Lernenden (4.4) betrachtet.

\subsection{Die Sicht der Schülerinnen und Schüler auf schülerseitiges Erklären und Begründen}

Den Analysen liegen 213 Sequenzen zugrunde, in denen die Schülerinnen und Schüler auf die Erklärungen und Begründungen ihrer beobachteten Peers Bezug nahmen. Es wurden neun unterschiedliche Felder thematischer Relevantsetzungen rekonstruiert, die sich drei Gruppen zuordnen lassen. Die erste umfasst Thematisierungen der inhaltlich-fachlichen Richtigkeit (4.2.1), die zweite Thematisierungen, bei denen diskursive Beiträge als Indikator für nicht unmittelbar Beobachtbares genutzt werden (4.2.2). Eine dritte Gruppe bilden schließlich Thematisierungen, bei denen diskursive Beiträge von Lernenden zu einem Beobachtungsgegenstand sui generis werden (4.2.3).

\subsubsection{Diskursive Beiträge in ihrer inhaltlich-fachlichen Richtigkeit}

Beim Betrachten der Videoclips setzen die Schülerinnen und Schüler häufig die inhaltliche Richtigkeit der beobachteten Erklärung bzw. Begründung relevant. Diese Art der thematischen Relevantsetzung fand sich auch in den Gruppendiskussionen der Lehrpersonen. Exemplarisch betrachten wir dazu Beispiel (6), 
das sich auf einen Videoausschnitt aus dem Deutschunterricht bezieht, in dem die Schülerin Feli den Begriff ,Lexikon“ in Abgrenzung zu ,Wörterbuch“ erklären soll. Ihre Erklärung lautete (s. auch Beispiel 1b oben):

dass man zum beispiel einen BUCHstaben hat, zum beispiel KA:, (.) und dann ist das äh einmal KLEIN und einmal GROSS geschrieben, =und da steht zum beispiel DIE katze daneben,und die ka (.) also katze (.) daneben SO, und DIE katze steht daneben als ähm (.) verb; $(4.0) \ll$ p $>$ SO weit war ich $>^{4}$

Beispiel (6) Sch-GD 1 (Jungen Gym, L1/L2), zu Feli

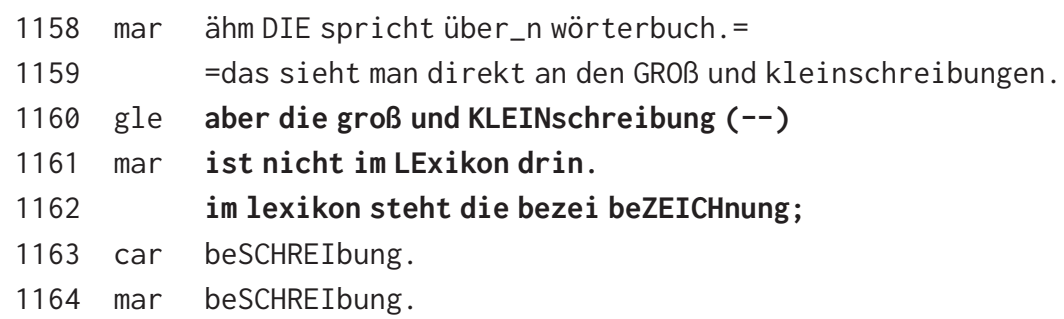

Markus trifft zunächst die Feststellung, dass sich Felis Erklärung auf das Wörterbuch, nicht aber auf den zu erklärenden Begriff ,Lexikon“ bezieht und somit inhaltlich unzutreffend ist. Als Beleg für Felis Irrtum führt er die Groß- und Kleinschreibung an, die sie als Definiens ins Feld geführt hatte. Glenn und Markus greifen die Frage nach der inhaltlichen Richtigkeit auf, indem sie richtigstellen, dass Felis Definiens nicht auf das Definiendum zutrifft (Z. 1160/61), und ein alternatives Definiens nennen (Z. 1162), das abschließend von Carl präzisiert wird (Z. 1163). Ebenso wie bei den Gruppendiskussionen der Lehrenden geht die Bewertung der inhaltlichen Richtigkeit hier mit einer Absicherung allgemeingültigen Fachwissens einher. Häufig werden dabei auch dichotome Kategorien wie richtig/falsch genutzt:

Beispiel (7) Sch-GD 5 (Mädchen, Ges, L1/L2), zu Feli

876 bar diese AUSsage von feli einfach nicht so richtig.

4 Die Transkriptausschnitte aus den Videoclips erscheinen als Zitate vor den jeweiligen Ausschnitten aus den Gruppendiskussionen. 
Festzuhalten ist, dass Bewertungen der inhaltlichen Richtigkeit in Form expliziter Feststellungen erfolgen und dabei fachliche Aspekte den Fokus bilden.

\subsubsection{Diskursive Beiträge als Indikator für nicht unmittelbar Beobachtbares}

In dieser zweiten Gruppe thematischer Relevantsetzungen werden diskursive Schülerbeiträge als Indikator für oder ,Fenster“ in nicht unmittelbar Beobachtbares genutzt: Von den Schülerbeiträgen wird entweder auf deren Verstehen, Identität oder emotionale und kognitive Befindlichkeit geschlossen.

\subsubsection{Indikator für schülerseitiges Verstehen}

Diese Art der thematischen Relevantsetzung, die sich auch bei den Lehrpersonen fand, ist insofern von der der inhaltlichen Richtigkeit abzugrenzen, als hier nicht der Inhalt einer beobachteten Schülererklärung oder -begründung den eigentlichen Fokus bildet, sondern diese lediglich als Anzeichen für oder ,Fenster' in das an sich nicht-beobachtbare Verstehen oder Wissen des Erklärenden genutzt wird. Das folgende Beispiel bezieht sich erneut auf die Erklärung von Feli (vgl. Beispiele 1b und 6 oben):

Beispiel (8) Sch-GD 3 (Jungen Ges low SES, L1 und L2), zu Feli

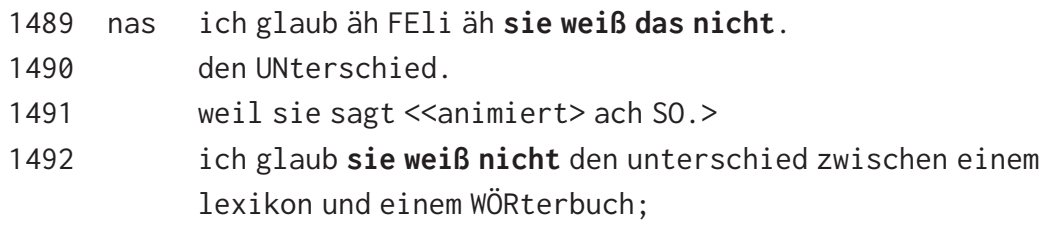

Den Fokus auf die Erklärerin macht Nasir hier schon durch den Fokusakzent auf dem Namen der beobachteten Schülerin erkennbar (Z. 1489). Mittels verba cogitandi werden Aussagen über deren Wissen getroffen (Z. 1489: „weiß nicht“, Z. 1492: „weiß das nicht“), die wiederum durch eine wörtliche Redewiedergabe begründet werden. Dabei wird Felis „ach SO“ als Dokument herangezogen, um Rückschlüsse auf ihr Wissen bzw. Verstehen zu ziehen. Dass es sich dabei um einen Deutungsprozess - die Interpretation also der an sich unbeobachtbaren Verstehensprozesse der Lernenden - handelt, wird vor allem durch solche verba cogitandi angezeigt, die die Mittelbarkeit bzw. den inferenziellen Charakter der eigenen Deutung markieren (Z. 1489/1492: „ich glaub“). 


\subsubsection{Indikator für schülerseitige Identität}

Die Schülerinnen und Schüler nutzen die beobachteten Erklärungen und Begründungen noch in einer anderen Hinsicht als Fenster in Nicht-Beobachtbares, indem sie in den Diskussionen identitätsbezogene Aspekte thematisch relevant setzen. Das folgende Beispiel bezieht sich auf einen Videoclip, in dem der Schüler Kostas im Rahmen einer mathematischen Rundungsaufgabe eine Begründung gibt:

${ }^{\circ} \mathrm{h}$ ja wenn man die drei_nsechzig ABrundet (.) auf die ZEHner, (-) dann kommt (.) dann isses (.) muss hinten IMmer eine null stehen, es MUSS sein, wenn_man RUNdet; und dann (.) da (-) wenn man (.) die drei WEGnimmt,=und die NULL dahinschiebt,=also (.) könnte man jetzt MAchen;=aber eigentlich is_es FALsch, (.) man muss einfach ABrunden, (-) und nä (.) nähere zahl mit ner NULL muss man dahin schreiben; (-)

Beispiel (9) Sch-GD 1 (Jungen Gym, L1/L2), zu Kostas

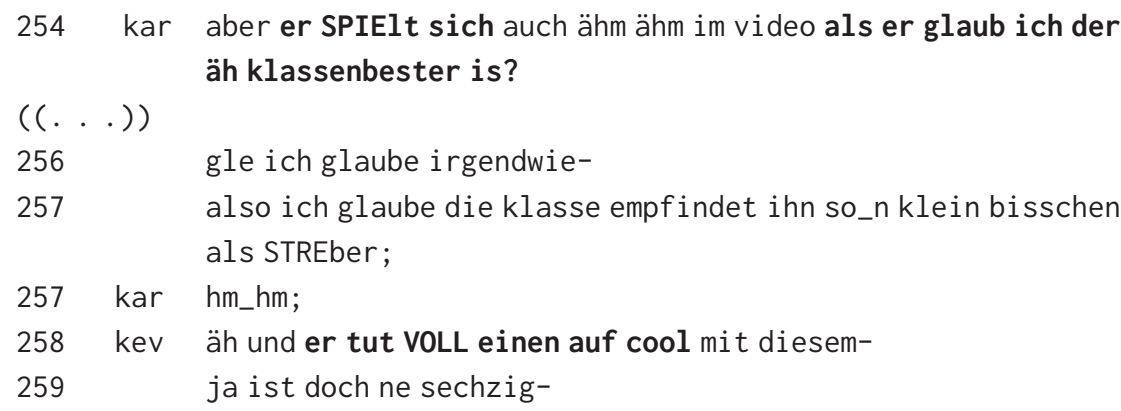

In ihrer Diskussion über Kostas‘ Begründung ziehen die diskutierenden Jungen einzelne kommunikative Verhaltensweisen (,SPIELT“, „tut VOLL einen auf cool“) als Dokument für eine bestimmte Identität („klassenbester“) heran. Diese wird als Selbstdarstellung gedeutet und mit Blick auf die soziale Kategorisierung durch die Mitschülerinnen und -schüler („STREber“) eingeschätzt. Beispiel (10) zeigt, dass soziale Kategorisierungen dabei keineswegs nur als Folge einer bestimmten Selbstdarstellung, sondern auch als deren Ursache gesehen werden:

Beispiel (10) Sch-GD 1 (Jungen Gym, L1/L2), zu Kostas

223 mar hm ich glaub dieser KOstas war auch ein ziemlicher (qua) oder er war halt halt so_n AUßenseiter;

$224 \quad$ und dann hat er sich versucht durch dieses RÄUspern am anfang ich glaub ein bisschen zu- (.)

225 ja WITZig zu machen; 


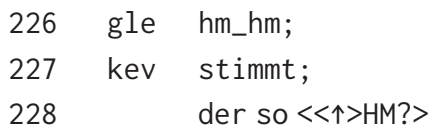

Markus sieht Kostas‘ sozialen Status als Außenseiter als Veranlassung für seine Inszenierung als „witzig“. Dabei wird Kostas“ Räuspern als Dokument für den Versuch gesehen, witzig erscheinen zu wollen. Den mittelbaren und inferenziellen Charakter seiner Deutung zeigt der beobachtende Markus durch entsprechende verba cogitandi (Z. 223, 224: „ich glaub“; s. auch Beispiel 9: Z. 254, 257) an.

Erklärungen und Begründungen werden somit von Schülerinnen und Schülern nicht nur als Fenster in das prinzipiell nicht-beobachtbare Verstehen, sondern auch in die Identität bzw. als Mittel der Selbstdarstellung genutzt. Während in den Gruppendiskussionen der Lehrpersonen durchgängig Metaphern aus dem Theater („er war ja auch so_n kleiner SCHAUspieler“; „und NUTZT das dann auch als bÜHne“) genutzt werden, mit denen eine implizite Relation zur eigenen institutionellen Rolle und Position (auf der Vorderbühne des Klassenraums) hergestellt wird, rücken die Schülerinnen und Schüler die soziale Rolle in der Klasse in den Vordergrund (s. a. Abschnitt 5.4 unten).

\subsubsection{Indikator für emotionale und kognitive Befindlichkeit}

Mit der Bezugnahme auf emotionale oder kognitive Befindlichkeiten nehmen Schülerinnen und Schüler eine thematische Relevantsetzung vor, die sich in den Gruppendiskussionen der Lehrpersonen nicht findet. Im Unterschied zu identitätsbezogenen Aspekten erfolgt dabei keine situationsübergreifende Kategorisierung, sondern eine Bezugnahme auf situativ und interaktiv hergestellte Phänomene. Beispiel (11) bezieht sich auf einen Videoausschnitt, in dem die Schülerin Petra im Anschluss an Feli (s. o.) den Unterschied zwischen einem Wörterbuch und einem Lexikon erklärt:

im LExikon da kann man ähm also wenn man da halt bei KA ähm katze hat, dann wird das erklärt woher die katze STAMMT und so; und beim WÖRterbuch da wird halt NUR, da kann man gucken wie es geSCHRIEben wird.

Beispiel (11) Sch-GD 1 (Jungen Gym, L1/L2), zu Petra

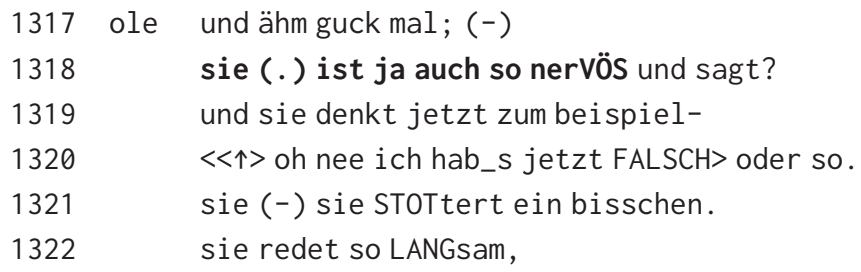


1324 gle die ist so ein kleines bisschen EINgeschüchtert wie der lehrer so ein kleines bisschen mit der.

1325 mar mit der mit dieser sache UMgeht?

Ebenso wie Verstehen und Identität sind auch emotionale und kognitive Befindlichkeiten nicht direkt beobachtbar, sondern können nur aus beobachtbarem Verhalten geschlossen werden. Dementsprechend lenkt Ole die Aufmerksamkeit der Mitdiskutierenden (,guck mal;“) auf Verhaltensweisen, die als Anzeichen („,stottert“, „redet so langsam“) für emotionale oder kognitive Zustände („nerVÖS“, „eingeschüchtert“) gedeutet werden können. Von dieser zunächst beobachtenden Außenperspektive wechselt Ole nun in eine Binnenperspektive, indem er eine Origo-Versetzung (Bühler 1934) in die beobachtete Person vornimmt und Petras Gedanken imaginiert (Z. 1319/1320: „sie denkt jetzt zum beispiel- . . . “). Die Redewiedergabe (Z. 1323), mittels der Ole Petras Verunsicherung inszeniert, dient keineswegs der Belustigung, sondern dazu, ihr Erleben für sich und andere nachvollziehbar $\mathrm{zu}$ machen. Dementsprechend zeigen Glenn und Markus ihr Verständnis an und führen Gründe für die Verunsicherung ins Feld. Diese werden nicht in der beobachteten Person selbst gesehen, sondern stets in einem verunsichernden Verhalten der Lehrperson (hier: „wie der lehrer so ein kleines bisschen mit der UMgeht“).

In den vorherigen Abschnitten wurden thematische Relevantsetzungen rekonstruiert, die sich auf Fachliches, auf Erschließungen identitäts- und wissensbezogener Aspekte sowie emotionaler bzw. kognitiver Befindlichkeiten im Zusammenhang mit den kommentierten Schüleräußerungen bezogen. Gegenstand der folgenden Abschnitte sind nun Relevantsetzungen sprachlich-diskursiver Aspekte.

\subsubsection{Diskursive Beiträge als Beobachtungsgegenstand sui generis}

An dieser dritten Gruppe thematischer Relevantsetzungen zeigt sich, dass die beobachtenden Schülerinnen und Schüler deutlich zwischen dem Verstehen (s. Abschnitt 4.2.1) und dem Versprachlichen unterscheiden. Neben Diskrepanzen zwischen dem Verstehen und Erklärenkönnen thematisieren sie auch Aspekte der Vertextung, Kontextualisierung, Markierung (Quasthoff, Heller \& Morek in diesem Band) sowie die Verständlichkeit von Erklärungen für andere Rezipienten als die Lehrperson. 


\subsubsection{Diskrepanz zwischen Verstehen und Erklärenkönnen}

Die Diskrepanz zwischen dem Verstehen und der Fähigkeit, das Verstandene auch sprachlich darstellen zu können, wird ausschließlich in den Gruppendiskussionen der Schülerinnen und Schüler thematisiert. Sie wird durchgängig auf die Gattung des Erklärens bezogen:

Beispiel (12) Sch-GD 4 (Jungen Ges, L2), zu Kostas

283 nur oder vielleicht KANN er ja abrunden.

284 aber er kann_s eben nicht richtig erKLÄren.

285 wal JA das seh ich auch so.

Beispiel (13) Sch-GD 5 (Mädchen Ges, L1/L2), zu Kostas

\section{4 fel manche leute KöNnen_s auch nicht erklären;}

315 auch wenn sie_s AUSrechnen.

Die Diskrepanz wird entweder spezifisch auf den im Video beobachteten Schüler bezogen (Beispiel 12) oder auch in verallgemeinerter Form thematisiert (Beispiel 13: „manche leute“). Dabei wird mittels gegenläufiger nuklearer Konturen oder Adversativjunktoren stets ein Kontrast zwischen dem fachlichen Können („KANN er ja abrunden“, „auch wenn sie_s AUSrechnen“) und dem Unvermögen des Erklärens hergestellt. Die Schülerinnen und Schüler nehmen damit eine bedeutsame Differenzierung vor: Zwar ziehen sie Erklärungen auch als Indikator für das schülerseitige Verstehen heran (vgl. Abschnitt 4.2.2.1); Erklärenkönnen und Verstehen sind aus ihrer Sicht aber dennoch nicht umstandslos gleichzusetzen, weil die sprachliche Darstellung zugleich als eine eigene, nicht immer ohne Weiteres $\mathrm{zu}$ bewältigende produktiv-sprachliche Anforderung wahrgenommen wird. Damit zeichnet sich im Vergleich zu den Lehrpersonen auf Seiten der Schülerinnen und Schüler eine differenziertere Einordnung sprachlich-diskursiver Anforderungen im Rahmen von Unterrichtsgesprächen ab.

\subsubsection{Vertextung}

Ein großer Anteil der Thematisierungen sprachlich-diskursiver Aspekte durch die Schülerinnen und Schüler bezieht sich auf die Vertextung. Damit ist eine Dimension von Diskurskompetenz (Quasthoff et al. 2019) angesprochen, die die interne Strukturierung explanativer bzw. argumentativer Äußerungspakete betrifft. Schülerinnen und Schüler thematisieren unterschiedliche Aspekte der Vertextung: erstens die Logik bzw. Ordnung des Aufbaus, zweitens den Umfang 
bzw. die Ausführlichkeit von Beiträgen, und drittens die genutzten Vertextungsverfahren (vgl. Heller in diesem Band), wie das Geben von Beispielen oder Vermeiden von Tautologien. Beispiel (14) bezieht sich auf einen Videoclip, in dem erklärt werden soll, was einen Geschäftsbrief im Unterschied zu einem persönlichen Brief auszeichnet. Die Erklärung des zweisprachigen Thasin lautete:

dass man DORT nicht so ähm (-) in dem inhalt nicht etwas von einem erlebnis oder so erzählt, SONde:rn (1.0) über (1.0) ähm (2.3) wie soll ich das jetzt SAgen; (1.8) zum beispiel über das geSCHÄFT das die ein euro macht;

Beispiel (14) Sch-GD 1 (Jungen Gym, L1/L2), zu Thasin

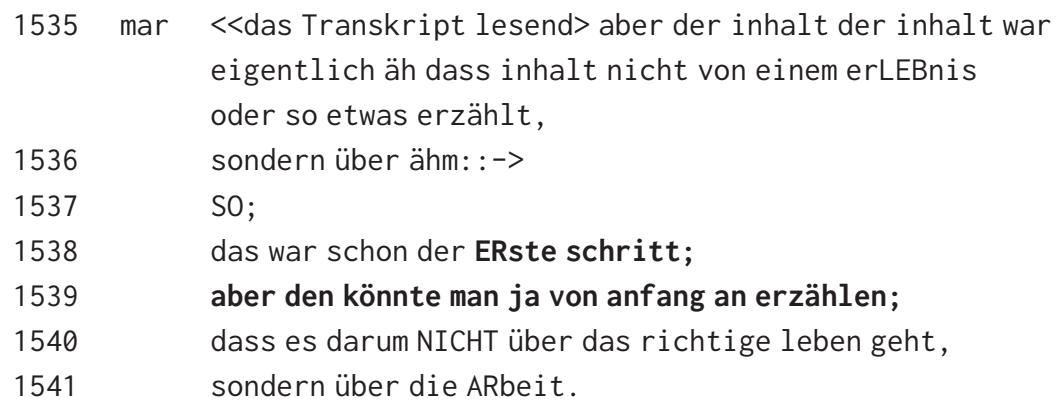

Markus liest bzw. paraphrasiert zunächst Thasins Äußerung (Z. 1535/36) und ordnet diesen Teil als ersten Schritt von Thasins Erklärung ein. Markiert durch den Adversativjunktor „aber“ (Z. 1539) formuliert er einen Alternativvorschlag und plädiert dafür, einen logischeren Ausgangspunkt zu wählen (,von anfang an erzählen"), den er paraphrasierend skizziert (Z. 1540/41). Markus richtet das Augenmerk damit auf das explanative Äußerungspaket als Ganzes, und zwar auf seine Logik und Ordnung im Aufbau.

Daneben wird auch häufig auf den Umfang von Erklärungen und Begründungen Bezug genommen. Das folgende Beispiel bezieht sich erneut auf Kostas (s. o.) und dessen Mitschülerin Katja, die auf knappe Art und Weise eine Regel zum Runden formuliert hatte.

Beispiel (15) Sch-GD 2 (Gym Jungen, L2), zu Kostas und Katja

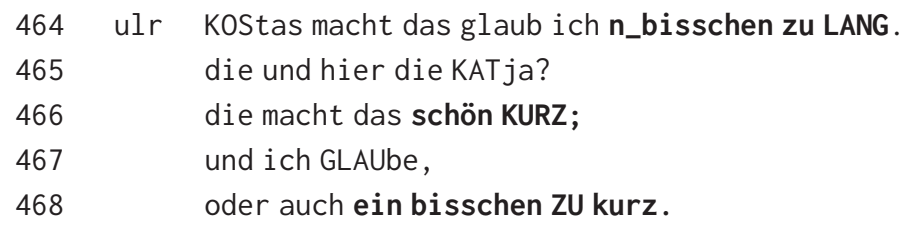


469

470

471

472

473

ähm ich finde eigentlich die machen_s GUT;

der INhalt ist gut,

aber (.) der (-) also die (.) GRÖße;

es ist $\ddot{H}$ bei KOStas ist VIEL,

und bei KATja ein bisschen weniger.

Ulrich produziert zunächst eine Bewertung des Umfangs der Erklärungen von Kostas und Katja („zu LANG“, „schön KURZ“), wobei er explizit eine Abgrenzung von inhaltlichen Gesichtspunkten vornimmt (Z. 470). Zugleich zeigt er durch verba cogitandi (,ich glaube“) und Heckenausdrücke (,eigentlich“) epistemische Unsicherheit an und wechselt schließlich von bewertenden zu rein deskriptiven Kommentaren (Z. 472/73). Inwiefern der Umfang einer Erklärung oder Begründung von Relevanz ist, wird von Ulrich nicht expliziert. Es lässt sich lediglich vermuten, dass der Umfang in Zusammenhang gesehen wird mit dem für den jeweiligen Kontext angemessenen Grad der Explizitheit oder auch Verständlichkeit.

Als ein dritter Aspekt der Vertextung werden in Beispiel (16) die Vertextungsverfahren (vgl. Heller in diesem Band) thematisiert, mittels derer eine Erklärung bzw. Begründung aufgebaut wird:

Beispiel (16) Sch-GD 6 (Mädchen Ges, L2), zu Kostas

389 til bisschen unerklär-

390 also ähm (.) nein nicht UNlogisch-

391 der erKLÄRT das nicht richtig.

$392 \operatorname{der}($.$) hm (.) der gibt dazu kein BEIspiel;$

393 zum beispiel DREI mal drei und dann sind neun-

$394 \quad$ und wie hat man das gerechnet und so?

Nachdem Tilbe das sie interessierende Phänomen der Vertextung zunächst ex negativo eingekreist hat („nein nicht UNlogisch“), stellt sie fest, dass „nicht richtig erKLÄRT“ wird. Aus dem Folgenden wird ersichtlich, dass sie sich damit nicht auf den Inhalt von Kostas Erklärung, sondern auf die Art und Weise der Vertextung bezieht. Eine Erklärung des Rundens verlangt aus ihrer Sicht das Geben eines Beispiels (Z. 392), was sie exemplarisch veranschaulicht (Z. 393), sowie das Vorführen des Vorgehens (Z. 394). Sie thematisiert damit zwei Vertextungsverfahren, die im Modus des veranschaulichenden Situierens (vgl. Heller in diesem Band) operieren und darauf zugeschnitten sind, komplexe oder abstrakte Operationen für die Rezipienten verstehbar zu machen. Das Erklären am Beispiel wird in den Gruppendiskussionen der Lehrkräfte ebenfalls angespro- 
chen, dort jedoch als typisch für ,Fünft- und Sechstklässler‘ und als zu überwindendes Übergangsphänomen kategorisiert (vgl. Heller, Quasthoff, Prediger et al. 2017). Damit deuten sich mit Blick auf die Vertextung divergierende Präferenzen von Lehrpersonen und Schülerinnen und Schülern an.

\subsubsection{Kontextualisierung}

In ihren Gruppendiskussionen thematisieren die Schülerinnen und Schüler auch Aspekte der Kontextualisierung (Quasthoff et al. 2019). Sie beziehen sich damit auf die diskursive Teilfähigkeit, eine Erklärung oder Begründung in den jeweiligen sequenziellen und sozialen Kontext einzupassen. In Beispiel (17) nimmt Dersin auf einen Videoclip Bezug, in dem die Schülerin Bea-Marie wie folgt auf die Frage der Mathematiklehrerin, ,wer fängt mal an das diagramm zu beSCHREIben;“" antwortet:

da werden tiere von dem alter wie ALT sie werden können ähm beschrieben;

Beispiel (17) Sch-GD 3 (Jungen Ges L1/L2), zu Bea-Marie

700 der also die lehrerin hat_s GUT am anfang gesagt .

701 könnt ihr mir das äh diagramm beSCHREIben.

702 dann die bea marie hat das irgendwie nIcht ausFüHRlich erzählt,

703 weil sie hat NUR gesagt,

704 da werden die tiere vom alter her; (-)

705 wie alt sie WERden können ähm beschrieben.

Der Blick des Beobachtenden ist hier auf die interaktive Sequenz vom lehrerseitigen Zugzwang bis zur Schülerantwort gerichtet. Der Fokus liegt darauf, inwiefern der schülerseitige Beitrag der mit der Frage der Mathematiklehrerin etablierten sequenziellen Erwartung - hier: dem globalen Zugzwang zum Beschreiben - entspricht. Dersin nutzt direkte und berichtende Redewiedergaben, um das nicht erwartungsgemäße sequenzielle Ineinandergreifen der Lehrer- und Schüleräußerungen zu demonstrieren und zeigt so, inwiefern hier die Kontextualisierung des Zugzwangs nicht gelingt. Er beurteilt den Zugzwang der Lehrerin zunächst als eindeutig bzw. „GUT“ (Z. 700) und verdeutlicht sodann zwei ganz zentrale Aspekte der Kontextualisierung: zum einen, dass Bea-Marie die Globalität des Zugzwangs nicht erkennt („nicht ausFÜHRlich“) und zum anderen, dass die mit dem Zugzwang angesteuerte Gattung verfehlt wird. Dabei wird die Gattung mit einem - zwar nicht gänzlich präzisen, aber dennoch den Kontrast zum 
Sagen/Nennen verdeutlichenden - Sprechhandlungsverb metasprachlich benannt („erZ̈̈HLT“ vs. „NUR gesagt“).

Aspekte der Kontextualisierung werden auch in den Gruppendiskussionen der Lehrpersonen thematisiert. Während letztere auf solcherlei Phänomene allerdings mittels kontrastiver Redewiedergabe lediglich implizit hindeuten, treffen die Schülerinnen und Schüler explizite Bewertungen, wenngleich diese mit Heckenausdrücken (,irgendwie“) als nicht ganz sicher markiert werden.

\title{
4.2.3.4 Markierung
}

Einen weiteren thematischen Rahmen der Bezugnahme auf diskursive Beiträge bilden sprachliche Formen und Ressourcen. Das folgende Beispiel bezieht sich auf einen Videoclip der Schülerin Neala, die im Deutschunterricht demonstriert, wie sie ein Interview mit dem Schulleiter ihrer Schule beginnen würde:

guten TAG ich bin die neala; und ich möchte GERne ein bisschen mehr über die sch die äh lehrer hier erfahren; und deshalb WILL ich ihnen ein paar fragen stellen;

Beispiel (18) Sch-GD 4 (Jungen Ges, L2), zu Neala

\author{
918 wal das will ist (. ) das $<<f>$ WILL $>$ ist ein bisschen UNhöflich; \\ 919 weil ich meine du kommst da REIN, \\ 920 äh sagst ich möchte ein bisschen über die LEHrer erfahren. \\ 921 deswegen $\langle<f>$ ! WILL! $>$ ich ihn ein paar fragen stellen. \\ 922 das macht ja den ganzen HÖFlichen (. ) eindruck kaputt.
}

Im Fokus von Walerios Kommentar steht nicht Nealas Äußerung als Ganzes, sondern lediglich eines ihrer Elemente (,will“), das hier mit Blick auf sozio-pragmatische Aspekte wie Höflichkeit bewertet wird. Dazu wird die Situation, für die Neala ihre Äußerung probehalber formuliert, reinszeniert: Walerio versetzt seine Zuhörer zunächst in das fiktive Szenario (Z. 919: „du kommst da REIN“) und animiert dann Nealas Äußerung mittels einer Redewiedergabe (Z. 920/921). Dies gibt ihm die Gelegenheit, das problematische Element besonders hervorzuheben, was hier durch den Fokusakzent und die Anhebung der Lautstärke («f > !WILL! >) erreicht wird. Auf diese Weise wird die unhöfliche Wirkung nicht nur behauptet, sondern auch wahrnehmbar gemacht, so dass die abschließende Bewertung (Z. 922) unmittelbar nachvollziehbar wird.

Mit dem Bezug auf die Nutzung sprachlicher Formen zur Herstellung von Kontextangemessenheit thematisieren die Schülerinnen und Schüler eine 
Dimension von Diskurskompetenz, die in der Diskurserwerbsforschung als Markierung (Quasthoff et al. 2019) bezeichnet wird. Während die Lehrpersonen in ihren Diskussionen mit Blick auf die Markierung vor allem die Präzision von Formulierungen, die syntaktische Vollständigkeit und lexikalische Repertoires ins Visier nehmen, beziehen sich die Schülerinnen und Schüler fast ausschließlich auf sozio-pragmatische Aspekte wie Höflichkeit und Respekt.

\subsubsection{Verständlichkeit für andere Rezipienten als die Lehrperson}

Die Verständlichkeit einer Schülererklärung oder -begründung für andere Rezipienten als die Lehrperson, d. h. vor allem für die Mitschülerinnen und -schüler, ist ein Aspekt, der in den Gruppendiskussionen der Lehrpersonen an keiner Stelle thematisiert wird. In Beispiel (19) bezieht sich ein Schüler erneut auf Kostas’ Begründung des Rundens (s. o.):

Beispiel (19) Sch-GD 4 (Jungen Ges, L2), zu Kostas

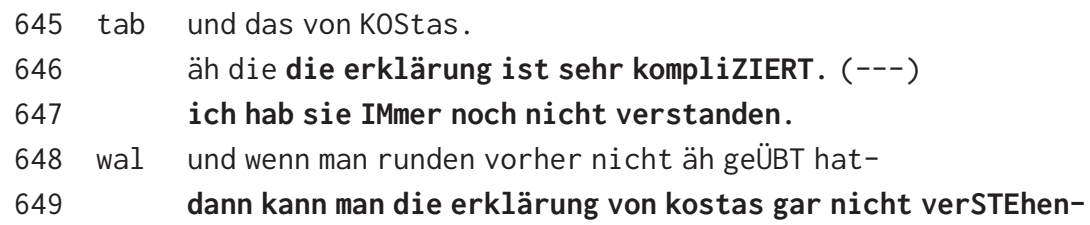

Tabito fokussiert die Begründung als Ganzes und bewertet sie als „kompliziert“. Diese Eigenschaft wird sodann asyndetisch als Begründung für das eigene Nichtverstehen (verbum cogitandi „verstanden“) herangezogen. Walerio ergänzt die Bewertung, indem er in verallgemeinernder Weise (Indefinitpronomen „man“) anfügt, dass das Verstehen von Kostas“ Begründung bereits eine Kenntnis des Gegenstands voraussetzt. Damit verweist er auf die originäre Funktion von Erklärungen und Begründungen, ein vonseiten des Rezipienten angezeigtes Verstehensdefizit zu beheben. Im schulischen Kontext tritt diese Funktion allerdings gegenüber der der Wissensdemonstration (Morek, Heller \& Quasthoff 2017) zuweilen in den Hintergrund: Häufig verfolgen Schülererklärungen und -begründungen vor allem den Zweck, der Lehrperson Einblick in den Stand des Verstehensprozesses bzw. der Wissensaneignung des/der Erklärenden zu geben. Im Unterschied dazu zeigt sich in den Gruppendiskussionen der Schülerinnen und Schüler, dass diese vor allem sich selbst als Rezipienten von Schülererklärungen und -begründungen sehen. 
Dies kommt auch in Beispiel (20) zum Ausdruck, in dem es um die Erklärung der Bedeutung des Begriffs ,Geschäftsbrief‘ (s. Beispiel 14 oben) geht:

Beispiel (20) Sch-GD 5 (Mädchen Ges, L1/L2), zu Denise

1324 kat also also deNIse verstehe ich nicht so ganz;

1325 tan denise meint glaub ich dass ein geSCHÄFTSbrief etwas geschäftliches ist;

1326 das ist jetzt eine erklärung die (man) nicht so richtig verSTEHen kann wenn-

1327 fel weil sich nicht jeder vorstellen KANN was ein geschäftlichen brief ist;

Auch hier wird von Katja zunächst subjektives Nichtverstehen konstatiert, das dann von Tanja generalisiert wird. Felicitas expliziert ergänzend, dass das Verstehen der Erklärung von Denise bereits erhebliche Vorkenntnisse voraussetzt. Aus Sicht der Schülerinnen und Schüler sind Erklärungen also dann gut und funktional, wenn sie an Wissensvoraussetzungen der Peers anknüpfen und so beschaffen sind, dass sie deren Verstehensprozess unterstützen. Damit wird hier von den diskutierenden Schülerinnen und Schülern erneut die Relevanz der Anschaulichkeit von Erklärungen (vgl. Abschnitt 4.2.3.2) hervorgehoben, während sie für Lehrpersonen vor allem ein Mittel der Wissensüberprüfung sind.

\subsection{Unterschiede zwischen Schülerinnen und Schülern mit Blick auf thematische Relevantsetzungen}

Gemäß der unterschiedlichen Zusammensetzung der Gruppen nach Sprachbiografie, sozio-ökonomischem Status und Geschlecht werden im nächsten Schritt häufigkeitsbezogene Unterschiede in den thematischen Relevantsetzungen dargestellt. Auf diesem Wege lässt sich feststellen, inwieweit die Relevantsetzungen kollektiv geteilt sind und „konjunktive Erfahrungsräume“ (Mannheim 1980: 73) reflektieren. Das Maß für die Erfassung der Häufigkeiten bildeten dabei thematische Einheiten $(n=213)$ anstelle von Turns, da sich Thematisierungen z. T. über mehr als einen Turn erstreckten.

Abbildung 1 zeigt die absoluten Häufigkeiten, um sichtbar zu machen, dass die Gruppen über ihre (vergleichbar langen) Diskussionen hinweg grundsätzlich unterschiedlich oft auf die beobachteten Schülererklärungen und -begründungen Bezug nehmen (zwischen 23 und 54 Thematisierungen). Die anschließenden Erläuterungen berücksichtigen die prozentualen Anteile. 


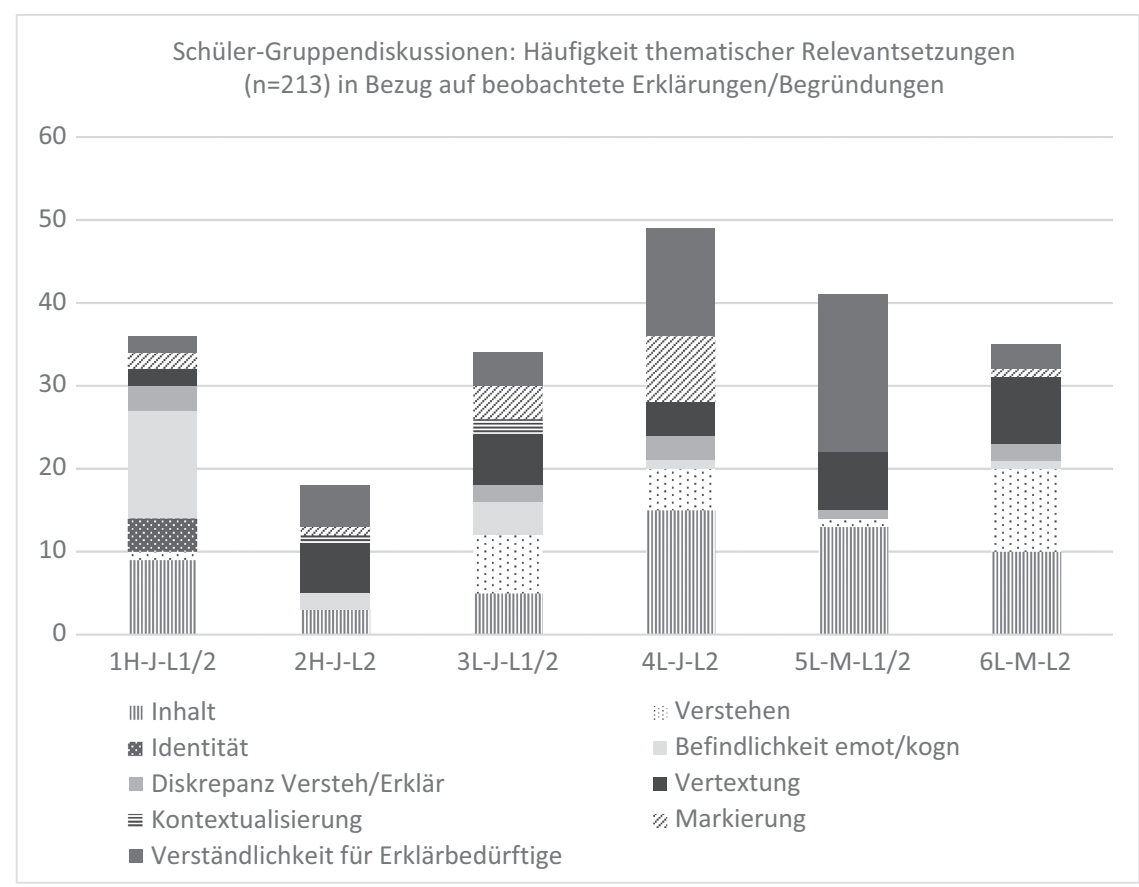

Abb. 1: Häufigkeit thematischer Relevantsetzungen in den Schüler-Gruppendiskussionen.

Unterschiede zeigen sich vor allem in Bezug auf das Merkmal des sozioökonomischen Hintergrunds der Kinder, nicht aber in Bezug auf die Merkmale Sprachbiografie und Geschlecht:

1. Schülerinnen und Schüler mit niedrigem sozio-ökonomischen Status (in unserer Studie: Gesamtschülerinnen und -schüler aus sozialen Brennpunkten) thematisieren im Vergleich zu den Schülerinnen und Schülern aus privilegierten Milieus häufiger die inhaltliche Richtigkeit der beobachteten Schülerbeiträge. Die Aushandlung darüber, was aus fachlicher Sicht zutreffend ist, beansprucht in diesen Schülergruppen mit durchschnittlich $26 \%$ aller Relevantsetzungen (gegenüber $15 \%$ bei den Gymnasialschülern) somit deutlich mehr Aufmerksamkeit und Kapazität. Diese Beobachtung ist insbesondere im Zusammenhang mit dem nächsten Befund interessant:

2. In denselben Gruppen macht die Verständlichkeit von Schülererklärungen für Erklärbedürftige im Durchschnitt ein Viertel (bei den Gymnasialschülerinnen und -schülern: $15 \%$ ) aller thematischen Relevantsetzungen aus. Sie sehen somit nicht die Lehrperson als alleinigen Adressaten einer Erklärung oder Begründung, sondern ebenso Rezipienten mit ,echtem‘ Erklärbedarf - 
also sich selbst. Dies lässt sich als Hinweis darauf verstehen, dass Schülererklärungen aus Sicht der Lernenden mit niedrigem sozio-ökonomischen Status nicht nur bzw. nicht primär die Funktion einer Wissensdemonstration gegenüber der Lehrperson haben, sondern auch eine Ressource für das Verstehen der Mitlernenden sind. Dies wird durch die Beobachtung gestützt, dass die Schülerinnen und Schüler durchgängig die Relevanz der Anschaulichkeit von Erklärungen hervorheben (vgl. Abschnitt 4.2.3.2 und 4.2.3.5).

3. Emotionale und kognitive Befindlichkeiten werden von Schülerinnen und Schülern mit hohem sozio-ökonomischen Status im Vergleich zu den Gesamtschülerinnen und -schülern aus sozialen Brennpunkten fünfmal häufiger relevant gesetzt, wobei zumeist Aspekte der epistemischen Verunsicherung durch die Lehrperson den Fokus bilden. Demgegenüber werden Schülererklärungen und -begründungen bei den sozial benachteiligten Kindern im Vergleich zu den Gymnasialschülern zehnmal häufiger als Fenster auf das schülerseitige Verstehen genutzt, wobei den Beobachteten i.d.R. ein Nichtverstehen attribuiert wird.

\subsection{Passungen und Divergenzen zwischen den expliziten Relevantsetzungen der Lehrpersonen und der Schülerinnen und Schüler}

Im Folgenden werden nun die expliziten Relevantsetzungen der Lehrpersonen (Heller, Quasthoff, Prediger et al. 2017) mit denen der Schülerinnen und Schüler verglichen, um Aufschluss über grundlegende Deutungsmuster und deren Passung zu gewinnen. $\mathrm{Zu}$ berücksichtigen ist, dass sich aus der handlungsentlasteten Diskussion über Unterricht nicht umstandslos Rückschlüsse auf die Wahrnehmungen im Unterricht ziehen lassen. Dennoch vermag der Vergleich erste Hinweise auf unterschiedliche Perspektiven auf das soziale Geschehen Unterricht zu geben.

Die Grundlage für den Vergleich bilden aufgrund der unterschiedlichen Anzahl von Lehrer- und Schüler-Gruppendiskussionen nicht die absoluten Häufigkeiten, sondern der prozentuale Anteil einer thematischen Relevantsetzung am Gesamt der Relevantsetzungen der jeweiligen Population (s. Abbildung 2).

Zunächst ist festzuhalten, dass die Schülerinnen und Schüler zwei thematische Relevantsetzungen vornehmen, die sich in den Gruppendiskussionen der Lehrpersonen nicht finden: Dies betrifft erstens die Verständlichkeit von Schülererklärungen und -begründungen für andere erklärbedürftige Adressaten, also vor allem für die Mitlernenden. Diese thematische Relevantsetzung macht $22 \%$ aller Vorkommen aus und kennzeichnet vor allem Schülerinnen und Schüler aus be- 


\section{Vergleich der prozentualen Anteile thematischer Relevantsetzungen in den Gruppendiskussionen der Lehrenden und Lernenden}

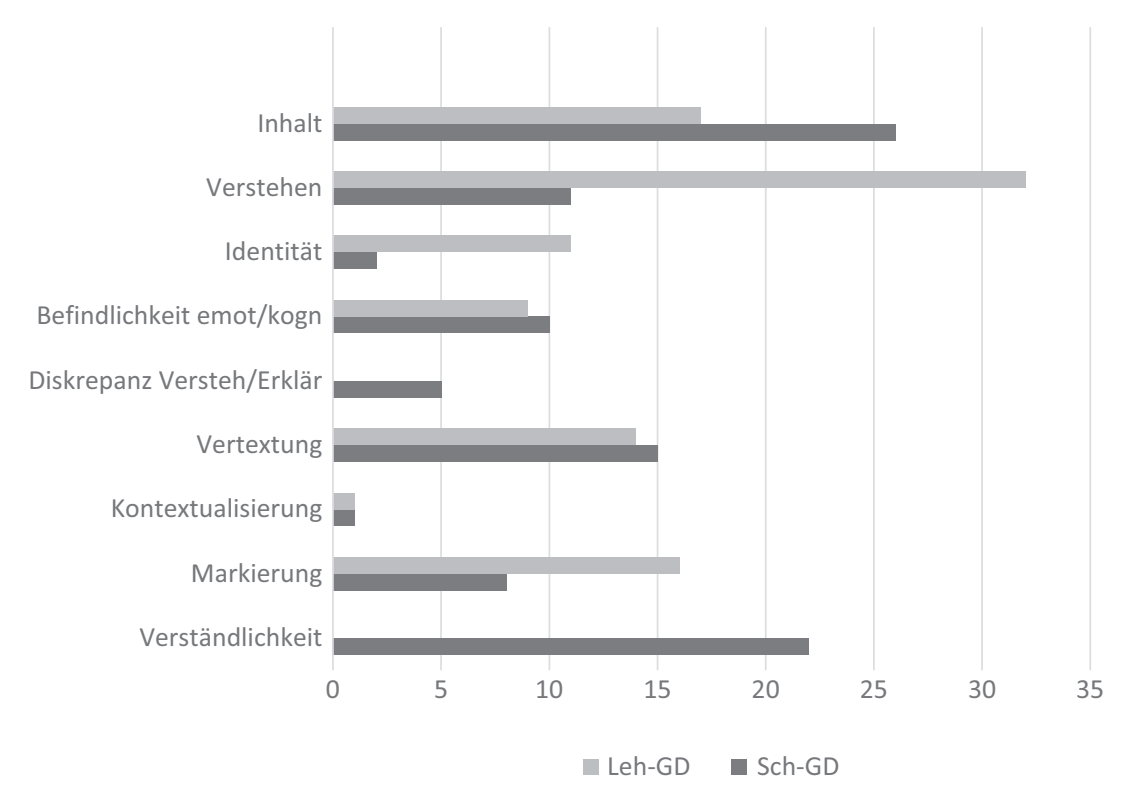

Abb. 2: Vergleich der Anteile der Relevantsetzungen von Lehrenden und Lernenden.

nachteiligten Milieus. Zweitens wird die Diskrepanz zwischen dem Verstehen und dem Nicht-Erklärenkönnen ausschließlich von den Lernenden thematisiert. Wenngleich der prozentuale Anteil mit $5 \%$ gering ist, findet sich diese thematische Relevantsetzung über unterschiedliche Gruppen hinweg und verweist darauf, dass die sprachlich-diskursive Darstellung komplexer Sachverhalte von den Schülerinnen und Schülern als eine Anforderung an sich wahrgenommen wird.

Betrachtet man nun, wie häufig diskursive Beiträge zu einem Beobachtungsgegenstand sui generis gemacht werden, so fällt überraschenderweise auf, dass dies in den Gruppendiskussionen der Schülerinnen und Schüler (51\%) deutlich häufiger geschieht als in denen der Lehrpersonen (31\%). Sprachlichdiskursive Aspekte sind somit für die Lernenden salienter als für die Lehrenden. Dem entspricht, dass die Lehrpersonen schülerseitige Erklärungen und Begründungen häufiger als Fenster in das schülerseitige Verstehen (32\% bei den Lehrpersonen vs. $11 \%$ bei den Lernenden) nutzen. Aus Sicht der Lehrpersonen erfüllt schülerseitiges Erklären und Begründen somit vor allem den Zweck, Aufschluss über das Verstehen bzw. Wissen des erklärenden bzw. begründenden 
Schülers zu erhalten. Dass der jeweilige Beitrag zugleich eine Ressource für das Verstehen der Mitlernenden darstellen kann, wird von ihnen ausgeblendet. Dies ist auch in dem Befund reflektiert, dass die Verständlichkeit für Erklärbedürftige von den Lehrpersonen an keiner Stelle thematisiert wird. Daran, dass die Fünftklässler sowohl die Verständlichkeit von Erklärungen und Begründungen häufig relevant setzen als auch eine anschauliche Vertextung einfordern, wird deutlich, dass schülerseitiges Erklären aus Schülersicht auch eine Ressource für den eigenen Verstehensprozess bilden kann. ${ }^{5}$

Hinsichtlich der Bezugnahmen auf die Kompetenzfacetten der Vertextung, Kontextualisierung und Markierung zeigen sich keine gravierenden Unterschiede: Sie machen bei den Lehrpersonen insgesamt $31 \%$, bei den Schülerinnen und Schülern $24 \%$ aus. Während die Lehrkräfte vermehrt auf die sprachliche Form fokussieren, werden Aspekte der Vertextung häufiger und in differenzierterer Weise (vgl. Abschnitt 4.2.3.2) von den Schülerinnen und Schülern thematisiert. Aus dem Umstand, dass Teilaspekte von Diskurskompetenz von Fünftklässlern und Lehrpersonen ungefähr gleich häufig angesprochen werden, lässt sich hier nun aber der Rückschluss ziehen, dass die Lehrpersonen (ebenso wie die Schülerinnen und Schüler) auf Alltagswissen und nicht etwa auf Professionswissen (Bromme 1992) über sprachlich-diskursive Phänomene zurückgreifen.

Der Vergleich macht sichtbar, dass Unterrichtsgespräche von den Beteiligten teils ähnlich, teils unterschiedlich perspektiviert werden. Sie stellen also für Lernende und Lehrende nur in Ausschnitten die gleiche „soziale Veranstaltung“ (Luckmann 1986) dar. Gerade für sprachliche Aspekte scheinen Schülerinnen und Schüler auf der Suche nach Hilfen zum Verstehen zuweilen sensibler zu sein.

Während wir dazu zunächst auf dem Wege der Rekonstruktion expliziter Relevantsetzungen einen Zugang entwickelt haben, betrachten wir nun in Abschnitt 5, welche impliziten Hinweise sich rekonstruieren lassen, die Aufschluss geben über das soziale Gefüge unter den Unterrichtsbeteiligten aus der Sicht von Lehrenden und Lernenden.

5 Wir interpretieren diesen Befund nicht als Hinweis darauf, dass Schülerinnen und Schüler die Institutionalität des Unterrichtsdiskurses verkennen und eher alltagsbezogene Verwendungen von Erklärungen erwarten. Vielmehr scheinen die Lernenden entsprechende verständnissichernde Funktionen des Erklärens gerade aus ihrer Schülerrolle heraus einzuklagen. 


\section{Zugänge zur impliziten Perspektivierung der sozialen Veranstaltung Unterricht: Referenzformen als soziale Kategorisierungen}

Wir beziehen uns in den in diesem Abschnitt vorgestellten Analysen theoretisch auf die membership categorization devices von Harvey Sacks $(1972,1979,1989)$. Sacks stellt in seinem später viel diskutierten und weiterentwickelten (z. B. Hausendorf 2000; Heller 2017; König 2014; Quasthoff 1978, 2017) Ansatz die Verbindung zwischen categorization devices (sozialen Kategorisierungen) und category bound activities (kategoriengebundenen Aktivitäten und Merkmalen) her. In Sacks Betrachtung ist diese Nutzung von (stereotypem) Alltagswissen über die typischen Merkmale (Yayyusi 1984) von sozialen Gruppen eine Ressource zur Herstellung von Verständigung. Members sortieren nach diesen Eigenschaften die Zugehörigkeit von Personen zu unterschiedlichen ,Abteilungen' im Ordnungssystem der sozialen Welt, so dass Schlussfolgerungen von Gruppenzugehörigkeit auf erwartbare Merkmale und umgekehrt ermöglicht werden.

Diese referentielle Mechanik lässt sich verdeutlichen an einem kleinen Test in Abwandlung des Beispiels von Sacks (1972): The baby cried. The mommy picked it up. In der abgewandelten Äußerung Das Baby schrie - die Schwester nahm es hoch wird der mehrdeutige Begriff, die Schwester von den meisten Befragten i. S. von Säuglings- oder Krankenschwester und nicht in der Bedeutung ,leibliche Schwester verstanden (Quasthoff 1978). Diese Art der Kohärenzherstellung kann mit der erwähnten Kopplung von category-bound activities an die mit der Kategorie aufgerufene Gruppenzugehörigkeit im Weltwissen erklärt werden: Das Kümmern um Babys gehört zu den kategoriengebundenen Tätigkeiten von Säuglings- und nicht unbedingt von leiblichen Schwestern. So dient dieses Alltagswissen als Ressource beim Verstehen der Referenzform, weil die beiden semantischen Lesarten nach Sacks auf unterschiedliche collections verweisen, d. h. auf unterschiedliche Einheiten im Gefüge der sozialen Ordnung, auf die implizit mit der Nennung der Kategorie ,Schwester' Bezug genommen werden kann: Im Fall der leiblichen Schwester und auch der Sack'schen Kategorie mommy - wird mit Rückgriff auf die Kollektion FAMILIE kategorisiert, im Fall der Säuglingsschwester könnte man von INSTITUTIONELLEN ROLLEN sprechen.

Wir finden hier also in den sprachlichen Ausdrücken zur personalen Referenzherstellung (,die Schwester', ,the mommy') sowie in den Folgen, die dies für die Kohärenz und Verständigung in Diskursen hat, eine implizite Nutzung eines Ordnungsgefüges der sozialen Welt, das die Sprechenden als members als geteilt unterstellen. Eine hearer's maxim erklärt nach Sacks die Verständigungs- 
mechanik des abgewandelten Beispiels mit der Kategorie ,Schwester‘: Wenn eine Kategorie mehreren Kollektionen angehören kann (FAMILIE und INSTITUTIONELLE ROLLE im Fall von ,Schwester') und in Verbindung mit einer kategoriengebundenen Tätigkeit genannt wird, die typisch für eine der categorization devices ist (hier: das Hochnehmen von Babies), wird die Kategorie i. S. dieser Kollektion verstanden (Sacks 1972: 337).

Soziale Kategorisierungen eröffnen somit durch die soziale Zuweisung in der personalen Referenzform einen Blick darauf, welchen Aspekt der sozialen Ordnung die Sprechenden im jeweiligen Kontext gerade relevant setzen: ,Das Kind‘, ,der Schüler‘, ,der Klassenclown` oder ,der Junge‘ können mögliche Referenzformen für dasselbe Individuum sein. Die jeweils für die Referenz genutzten Kategorien unterscheiden sich jedoch hinsichtlich der Kollektionen in der sozialen Welt, auf die sie implizit verweisen: STAGE OF LIFE (Sacks 1972), INSTITUTIONELle Rolle, Rolle IN DER KLASSE ALS PEERGROUP oder GESCHLECHT.

Insofern als jede realisierte Referenzform als eine Entscheidung gegen eine andere mögliche gesehen werden kann, ist die mit der Referenzform vorgenommene implizite Relevantsetzung auch unter kontextuellen Bedingungen zu betrachten. Diese Semantik des (sozialen) Weltwissens verweist auf einen Aspekt der kohärenzbildenden Kraft der personalen Referenzformen, der über die von Sacks beschriebene Kopplung zu den kategoriengebundenen Merkmalen hinausgeht. Wenn man sowohl die alternativen Kollektionen als auch die übrigen Elemente der jeweils aufgerufenen Kollektion als einschlägiges Alltagswissen der Beteiligten einbezieht, ist zu erkennen, welchen weiteren Beitrag die Referenzformen beim Aufbau von Kohärenz leisten: Die Bezeichnung eines Individuums oder einer Gruppe als ,Kind(er)' etwa sieht im gegebenen Kontext davon ab, die Bezeichneten als Teil des professionellen Kontextes Schule (,Schüler`) zu perspektivieren oder ihr Geschlecht relevant zu setzen (,Mädchen‘/,Jungen'). Mit der Bezeichnung als ,Kind' im Rahmen der Kollektion LEBENSPHASE wird das benannte Individuum (oder die Gruppe) implizit in Zusammenhang gebracht mit Erwachsenen, Alten etc. Die Bezeichnung ,Schüler ${ }^{\star}$ würde stattdessen mit der Kollektion InSTITUTIONELLE RoLLE (in der Schule) die Elemente dieser Kollektion (,Lehrer‘, ,Schulleiter‘ etc.) aufrufen. Die Kollektion ROLLE IN DER KLASSE ALS PEERGROUP (,Klassenclown') wiederum würde auf ein Ordnungssystem mit alternativen Besetzungen wie ,Streber‘, ,Schleimer` o. Ä. verweisen.

Diese auf dem sozialen Weltwissen basierenden kohärenzbildenden Mechaniken machen soziale Kategorisierungen als Analysezugang für die Gruppendiskussionen im vorliegenden Zusammenhang methodisch einschlägig: Wir sind interessiert an der Rekonstruktion gerade auch solcher Indikatoren für die alltagsweltliche Ordnung der sozialen Umgebung aus der Sicht der Lernenden und Lehrenden, die von den Teilnehmenden der Gruppendiskussionen nicht 
explizit inhaltlich thematisiert werden (s. o. Abschnitt 1). Wir finden derartige Indikatoren durch die Aufdeckung der mit den sprachlichen Referenzformen implizit relevant gesetzten Gefüge sozialer Ordnung im Rahmen der membership categorization devices, die sowohl aus den ausgewählten Kollektionen als auch den kategoriengebundenen Zuschreibungen rekonstruierbar werden. Im vorliegenden Beitrag konzentrieren wir uns auf die Analyse der nominalen sozialen Kategorisierungen in Verbindung mit den zugehörigen Kollektionen und ihrer Häufigkeitsverteilung im Vergleich zwischen den verschiedenen Gruppen der Lehrenden und der Lernenden sowie zwischen Lehrenden und Lernenden (zu einer stärker auf die category-bound activities fokussierenden Analyse s. Heller \& Quasthoff 2020).

Aus dem bisher Gesagten ist abzuleiten, dass die Wahl der jeweiligen sozial kategorisierenden Referenzform trotz ihrer Implizitheit nicht beliebig sein sollte, sondern durchaus etwas zum Ausdruck bringt, auf das es Sprechenden im jeweiligen Kontext ankommt. Dies lässt sich in unseren Daten auf verschiedene Weise belegen, etwa am Fall einer Selbstreparatur im folgenden Beispiel:

Beispiel (21) L-GD 2 (Ges, ML/DL); Kontext: Vermeidung von Fachtermini

0805 rie und (. ) n und sagt man nur DEShalb?

0806 damit die schü ah kinder WISsen-

0807 was das eigentlich äh beDEUtet.

Hier wechselt die sprechende Lehrkraft von der collection INSTITUTIONELLE ROLLE (,Schüler') zu LEBENSPHASE (,Kinder`) und indiziert damit, dass ihr in diesem Kontext die Einordnung in ein außerschulisches Ordnungssystem wichtig ist. Weitere Hinweise zur nicht austauschbaren Perspektivierung der Kollektionen finden sich in den Beispielen (23-25) unter 5.2 unten.

\subsection{Vorgehen in der Analyse}

Zur Abgrenzung der interessierenden Phänomene bei der Sichtung der Daten nutzen wir die Tatsache, dass sich die Sacks'sche Unterteilung in soziale Kategorien und kategoriengebundene Tätigkeiten aus linguistischer Sicht satzgrammatisch beziehen lässt auf den Zusammenhang von Nominal- und Verbalphrase bzw. funktional auf den von Referenz und Prädikation. Zu den Nominalphrasen gehören entsprechend im grammatischen Sinn nicht nur Nomen und ihre Determinatoren (,die Schüler'), sondern auch komplexe 
Nominalphrasen, z. B. solche mit Erweiterung durch Relativsätze (,die (Kinder), die Deutsch nicht als erste Sprache haben').

Den Analysen liegt ein Teilkorpus der vollständig transkribierten Gruppendiskussionen zugrunde, das die Sequenzen enthält, die inhaltsanalytisch als relevant für soziale Kategorisierungen kodiert wurden und sich auf Schülerinnen und Schüler beziehen.

Insgesamt ist bei den zu berichtenden Beobachtungen zu den personalreferentiellen Kategorisierungen natürlich $\mathrm{zu}$ beachten, dass der durch die Video-Stimuli und die Gesprächsführung gegebene Kontext der Gruppendiskussionen die Art der sozialen Kategorisierungen und der damit verbundenen Kollektionen steuert; in einem spontanen Gespräch auf dem Schulhof/im Lehrerzimmer würden möglicherweise andere Kategorien genutzt. Allerdings wurde in der Erhebung der Gruppendiskussionen - wie in Abschnitt 3 oben dargestellt weitgehend von einer inhaltlichen Steuerung abgesehen, so dass die beteiligten Lehrpersonen und Schülerinnen und Schüler im Rahmen des Redens über die Videoausschnitte weitgehend ungesteuert waren. Da der Kontext der Video-Stimuli und des Diskussionsformats zudem für alle Gruppen der gleiche war, sind gerade aus den Vergleichen, auch im Hinblick auf Häufigkeiten, durchaus aufschlussreiche Schlussfolgerungen (s. u. Abschnitt 5.4) zu ziehen.

Um nun auch mit Hilfe dieses zweiten analytischen Zugangs einen Einblick in die Passungen und Divergenzen der sozialen Deutungsmuster (Bohnsack 2010) sowohl unter verschiedenen Lehrkräften und verschiedenen Lernenden als auch im Vergleich zwischen Lehrenden und Lernenden zu erhalten, werden die Vorkommen zu den einzelnen Kollektionen in den folgenden Abschnitten 5.2 und $5.3 \mathrm{zu}$ den Lehrer- und Schüler-Diskussionen zunächst beschrieben und sodann quantifiziert. Die relativen Häufigkeitsverteilungen der sprachlich realisierten Kategorisierungen geben auch einen Hinweis darauf, wie verbreitet die impliziten Relevantsetzungen entsprechender Aspekte in den Perspektivierungen des sozialen Gefüges jeweils sind, d. h. inwieweit sie als kulturell geteilt erachtet werden können.

Dabei betrachten wir ausschließlich die kategorisierenden Bezugnahmen der Diskutierenden auf Schülerinnen und Schüler: I.S. des eingangs (s. o. Abschnitt 1) erwähnten Anwendungsbezugs interessiert uns hier insbesondere, wie ähnlich oder unterschiedlich die soziale Gemeinschaft von Schulklassen perspektiviert wird, um langfristig aus den entsprechenden Passungen oder Divergenzen Rückschlüsse auf die Partizipationschancen und die Partizipationsbereitschaft von Schülerinnen und Schülern an der sozialen Veranstaltung Unterricht ziehen zu können. 


\subsection{Die impliziten Relevantsetzungen von Lehrenden}

Eine induktive Analyse aller Vorkommen von nominalen sozialen Kategorisierungen (im Folgenden: NSK) in den Lehrenden-Gruppendiskussionen mit Bezug auf Schülerinnen und Schüler ergab die folgenden Kollektionen, auf die implizit durch die Nennung zugehöriger Kategorien verwiesen wurde:

- LEBENSPHASE (,Kinder')

- INSTITUTIONELLE ROLLEN (,Schüler‘, ,Fünftklässler`)

- Leistungsvermögen (,die Fitten', ,die Dummen‘, ,die Schwachen` etc.)

- SOZIALES MILIEU (,Schüler mit Migrationshintergrund', unsere Klientel‘, ,Kinder aus verschiedenen Grundschulen/Förderschulen')

- psychologische Kategorien (,cooler Typ')

- FAMILIE $^{6}$ (,Vater', ,Mutter', ,Bruder $)^{7}$

Zur Illustration der Verwendung entsprechender Kategorien im Kontext der Gruppendiskussionen zeigen wir im Folgenden typische Sequenzen zu den einzelnen Kollektionen, aus denen sich der Zusammenhang zwischen den kategorisierenden Referenzformen und den assoziierten Kollektionen erschließen lässt.

Ein prototypischer Verweis auf die Kollektion LEBENSPHASE findet sich im folgenden Beispiel (22):

Beispiel (22) L-GD1 (Gym, ML/DL)

324 kle und äh in dem alter ist es ja auch oft so?

325 dass ähm das begründen jetzt NICHT so-

326 also wenn man nem KIND sagt?

327 es IST so?

328 wenn die noch sehr KINDlich sind?

329 dann IST es für den so;

Der Mathematiklehrer eines Gymnasiums nimmt hier Bezug auf Entwicklungsphasen und verwendet folgerichtig die Kategorie ,Kind', die die Kollektion LEBENSPHASEN aufruft. In diesem inhaltlichen Kontext, in dem „KIND“ (Z. 326)

\footnotetext{
6 FAMILIE gehört im strengen Sinn nicht in das Korpus von NSK von Schülerinnen und Schüler, wurde aber erfasst, um die Rolle abzubilden, die das private Umfeld aus der Sicht der Sprechenden jeweils spielt.

7 Für die Gruppendiskussionen unter Schülerinnen und Schülern (s. u. Abschnitt 4.3) wird diese Liste erweitert um die Kollektion: ROLLE IN DER KLASSE ALS PEER GROUP (,Außenseiter', ,Beliebter‘, ,Streber‘, ,einer, der nicht immer aufpasst').
} 
klar als Typisierung einer bestimmten Lebensphase (Z. 324: „in dem alter“, Z. 328: „noch sehr KINDlich“) zu hören ist, wäre die alternative Benennung mit ,Schüler' und damit der implizite Verweis auf die INSTITUTIONELLE ROLLE in Z. 326 nicht stimmig.

Ähnlich klar erfolgt demgegenüber im nächsten Beispiel eine Typisierung mit Bezug auf die INSTITUTIONELLEN ROLLEN in Klassenzimmer seitens einer Gymnasiallehrerin:

Beispiel (23) L-GD 1 (Gym, ML/DL)

1668 wal was soll denn jetzt auch noch weiter KoMmen?

1669 also was soll man denn als schüler noch weiter SAgen?

Hier ist die implizit mit aufgerufene Kontrastkategorie innerhalb der Kollektion eindeutig die des ,Lehrers‘, denn es geht um die Art, wie Lehrpersonen Schülerbeiträge initiieren. Der auch sprachlich als Typisierung markierte Ausdruck „als schüler“ in Z. 1669 ist also auch hier nicht austauschbar etwa mit ,Kind“. Dies wird besonders nachvollziehbar, wenn man das folgende Beispiel betrachtet, das ebenfalls die typisierende Form ,als + Kategorienname‘, diesmal jedoch wiederum im Rahmen der Kollektion LEBENSPHASE nutzt:

Beispiel (24) L-GD 1 (Gym, ML/DL)

1660 kle müsste ja eigentlich-

1661 so nach ner halben stunde als kind würd ich tot UMfallen.

1662 und DENken-

1663 <<animiert> boa ist das HIER wieder stressig.>

Auch hier wird die Relevantsetzung in Form der Kollektion LEBENSPHASE im Unterschied zur INSTITUTIONELLEN ROLLE trotz ihrer Implizitheit besonders deutlich: Der Lehrer inszeniert die Typisierung ,als kind“ (Z. 1661), indem er die kindliche Ungeduld hyperbelhaft anspricht („tot UMfallen“) und sogar in direkter Rede (Z. 1663) eine kindliche Ausdrucksweise animiert. ${ }^{8}$

8 Diese sehr deutliche Systematik im Umgang mit der Varianz zwischen den Kollektionen INSTITUTIONELLE ROLLE und LEBENSPHASE scheint interessanterweise auf die hier zugrundeliegenden Korpusausschnitte begrenzt zu sein, die auch inhaltsanalytisch der Rubrik soziale Kategorisierung zugeordnet wurden (s. o. 5.1). Bei späterer Durchsicht des Gesamtkorpus der 
Eine typische Bezugnahme auf die Kollektion LeISTUNGSVERMÖGEN findet sich im folgenden Beispiel einer expliziten Kopplung zwischen Kategorie und kategoriengebundener Eigenschaft:

Beispiel (25) L-GD 1 (Gym, ML/DL)

2277 kle abSTRAKT ist sowieso immer schlecht.

2279 also schwierig für die DUMmen.

Im Lichte des oben Gesagten ist interessant, dass Qualifizierungen nach LEISTUNGSVERMÖGEN in Nominalphrasen mit attributiven Adjektiven (fit, schwach, dumm) im zugrundeliegenden Korpus typischerweise in Kombination mit der Kategorisierung ,Kinder‘ und nicht ,Schüler' auftauchen:

Beispiel (26) L-GD 2 (Ges, ML/DL)

2689 les ich arbeite mit einer kollegin die ganz bewusst auch die FITten kinder (so lockt)?

2690 sie kommt also hauptsächlich aus der Oberstufe?

$((.)$.

2712 weil sie einfach nur mal ZEIgen wollte?

2713 äh dass die fitten?

2714 weil die haben das dann ( );

2715 weil die haben sehr fitte kinder?

In diesem Kontext der Einordnung nach Leistungsvermögen wäre die Kategorie ,Schüler“ möglicherweise sogar eher erwartbar als „kinder“ („Oberstufe“). Nach dem oben Gesagten könnte man aus der in gewisser Weise überraschenden Wahl der Kollektion LEBENSPHASE mit aller Vorsicht schlussfolgern, dass dahinter die alltagsweltliche Annahme aufscheint, ,fit‘ oder ,dumm' seien die entsprechenden Individuen ,als Kinder' auf Grund ihres außerschulisch bedingten Entwicklungsstandes und nicht auf Grund ihrer unterrichtlichen Förderung ,als Schüler‘. Der größere Zusammenhang der gezeigten Sequenz

\footnotetext{
Gruppendiskussionen in einem anderen Analysezusammenhang fanden sich auch Fälle, in denen Kind und Schüler im jeweiligen Kontext mehr oder weniger austauschbar verwendet wurden. Neben der hier beschriebenen Systematik scheint also auch die jeweilige Präferenz von Sprecherinnen und Sprechern eine Rolle zu spielen, insofern als manche der Diskutierenden grundsätzlich eher dazu neigen, die Lernenden nach LEBENSPHASE als ,Kinder` oder institutionell als ,Schüler‘ zu kategorisieren.
} 
unterstützt diese Lesart deutlich: Vorausgehend ist in der Diskussion davon die Rede, dass man manchmal überrascht über die Leistungsfähigkeit Einzelner ist, und im Folgekontext äußert die Lehrerin: „also ich denk die kommen aus nem elternhaus? wo also auch sprache ne ROLle spielt.“ (Z. 2701f).

Das folgende Beispiel zeigt gleich mehrere Merkmale der impliziten Relevantsetzung des SOZIALEN MiliEus der Schülerinnen und Schüler:

Beispiel (27) L-GD 2 (Ges, ML/DL)

0955 les das hängt ganz stark von den GRUNDschulen ab.

0956 und zwar von der klienTEL der grundschulen.

0957 also das kann man ganz ähm-

0958 es gibt KLASsen?

0959 die das selbstverSTÄNDlich machen?

0960 auch DEUTSCH mit den fachbegriffen.

0961 es gibt KLASsen?

0962 ähm wo die kinder mit nem hohen anteil an migration ähm migrationshintergrund erstmal versuchen über die DEUtschen begriffe sachen zu klären.

Der Ausdruck ,Klientel' (vgl. auch Beispiel 4 oben) kann in unseren Daten als eine Art Euphemismus für ,Leistungsschwache‘ angesehen werden:

Beispiel (28) L-GD 2 (Ges, ML/DL)

2830 wir MÜSsen unserer klientel gerecht werden.

2831 wir haben so viel schülerinnen und schüler die wörter wie GESCHÄFTlich nicht verstehen.

2832 SACHlich nicht verstehen.

Beispiel (29) L-GD 2 (Ges, ML/DL)

0481 also wenn ich an unser klienTEL denke?

0482 das ist eine sehr UNdeutliche aufgabenstellung.=ja?

0483 also das ist (. ) wischi WAschi so_n bisschen.

Wenn die Sprecherin in Beispiel (27) oben ihren Verweis auf ,Grundschulen“ (Z. 955) eigens spezifiziert als: „klienTEL der grundschulen“ (Z. 956), dann kann das als versteckter Hinweis auf die soziale Zusammensetzung des Einzugsbereichs der jeweiligen Grundschule verstanden werden, insbesondere, 
weil der Ausdruck typischerweise - wie auch in den Beispielen (28) und (29) im inhaltlichen Zusammenhang mit nicht-muttersprachlichen Schülerinnen und Schülern vorkommt. Die Referenzform „(unsere) klienTEL“ bleibt dabei semantisch jedoch unbestimmt hinsichtlich der damit verbundenen sozialen Einordnung. I.d.S. kann die semantische Unterbestimmtheit im Übrigen als Verfahren betrachtet werden, wie Sprechende die Geteiltheit des zugrundeliegenden Wissens indizieren. Die inhaltlichen Folgerungen aus der Kategorisierung „(unser) klienTEL“ werden offenbar in der Profession für so selbstverständlich gehalten, dass sich jede Explikation erübrigt.

Bei der Kategorisierung des Status von Schülerinnen und Schülern mit Deutsch als zusätzlicher Sprache fällt unter sprachlichen Gesichtspunkten durchgängig auf, dass die Sprecher durch unterschiedliche Arten von Verzögerungen eine besondere Sorgfalt bei der Benennung der Kategorie inszenieren: Der beobachtbar hohe Formulierungsaufwand (Selbstreparaturen, syntaktisch komplexe Referenzformen, prosodische Verzögerungsphänomene etc. (vgl. auch Beispiel 27, Z. 962)) bei der kategorialen Diskriminierung gerade dieser Schülergruppe könnte darauf hindeuten, dass man sich im professionellen Diskussionsprozess unter Kollegen nicht dem Verdacht des Diskriminierungsdiskurses i. S. der sprachlichen Ausgrenzung und Abwertung aussetzen möchte:

Beispiel (30) L-GD 2 (Ges, DL/ML)

0970 aber wenn ich jetzt KINder habe;

0971 die deutsch nicht als-

0972 äh na SACH schon-

0973 ERste sprache haben,

0974 die haben probleme DAS was ich als lehrer so sage,

0975 zu verSTEhen;

Hier wird eine besonders hohe Kontrolle bzw. Unsicherheit („na SACH schon“) bei der ,politisch korrekten' Bezugnahme sprachlich inszeniert: Eine NSK, die nach den Mechanismen der Herstellung von Referenz auf Personen (Sacks \& Schegloff 1979) sprachökonomisch naheliegend wäre, wäre z. B. ,Ausländerkinder‘. Diese noch bis in die 80er Jahre durchaus übliche Referenzform steht aber inzwischen unter Diskriminierungsverdacht - eine professionell eingeführte und unverdächtige alternative einfache Kategorisierungsform steht den Lehrkräften offenbar nicht zur Verfügung.

Die diskutierten Sequenzen konnten also die oben beschriebenen Mechanismen des Aufrufens impliziter Kontexte durch die Wahl der einer bestimmten Kollektion zugehörigen Referenzform im Zusammenhang vorführen: Der Verweis 
auf die LEBENSPHASE durch die NSK, Kind“ transportiert oft unausgesprochen die Konnotation, dass Lernende durch außerschulisch gegebene Umstände positiv oder negativ geprägt sind, gerade auch in ihrem LEISTUNGSVERMÖGEN als ,Fitte“. Der Verweis auf das SOzIALE Milieu, besonders verbunden mit der Referenzform ,(unsere) Klientel', beleuchtet die soziale Herkunft primär unter dem Aspekt der Benachteiligung bzw. der besonderen Anforderungen an das Lehrerhandeln. Einen Sonderfall in diesem Zusammenhang stellen Verweise auf nicht-muttersprachliche Lernende dar, für deren Benennung ein auffälliger sprachlicher Aufwand betrieben wird. Die Benennung der Lernenden als ,Schüler hingegen präsentiert sie in ihrer INSTITUTIONELLEN ROLLE als Gegenpart zu Lehrpersonen und als Objekt ihres professionellen Handelns.

Nachdem wir das analytische Potenzial der NSK im Kontext der Beispiele beschrieben haben, wenden wir uns im Folgenden ihrer Verteilung in den unterschiedlichen Diskussionsgruppen zunächst der Lehrenden zu. Wie oben erwähnt (Abschnitt 3) bildete die Zusammensetzung der jeweiligen Diskussionsgruppen die in der Unterrichtsstudie von InterPass untersuchten Schulformen und Fächer ab, um Unterschiede in den jeweiligen gruppenbezogenen Relevantsetzungen bei der Konfrontation mit denselben videographierten Unterrichtssequenzen in ihrer möglichen sozialen Systematik verstehen zu können. Die Verteilungen der Kollektionen zwischen den Gruppen von Gymnasial- bzw. Gesamtschul- und Deutsch- sowie Mathematiklehrkräften wird in Abb. 3 unten graphisch gegenübergestellt.

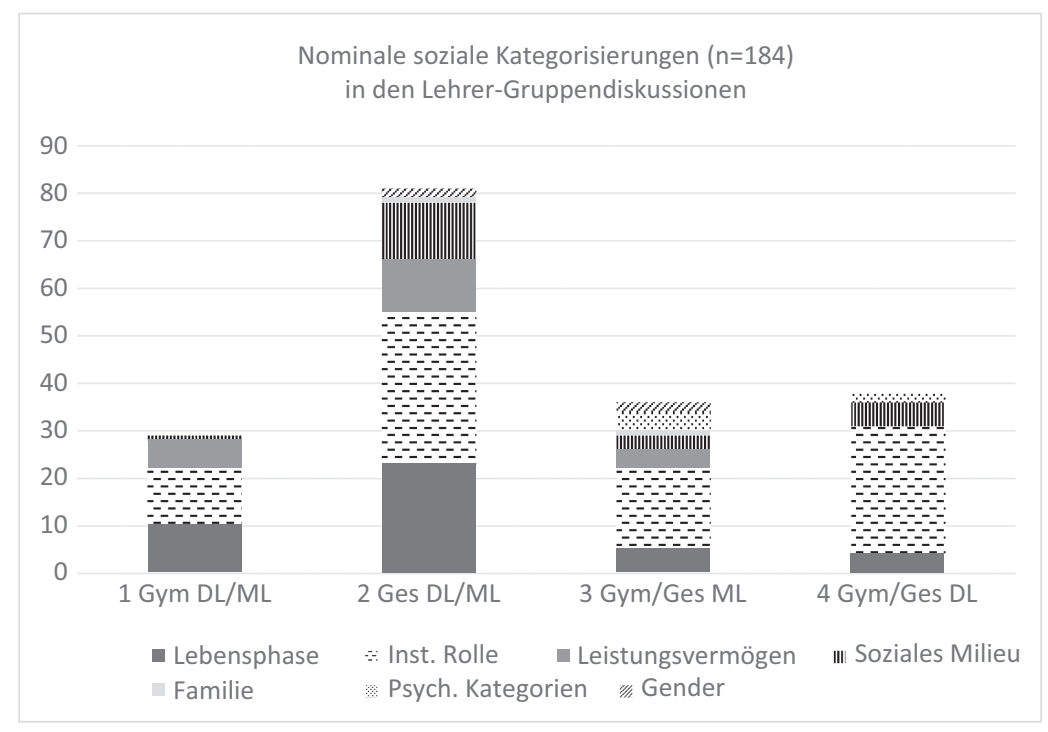

Abb. 3: Kollektionen in den Lehrer-Gruppendiskussionen. 
Um die absoluten Zahlen der Vorkommen von NSK nach ihrem relativen Gewicht einordnen zu können, wurden sie bezogen auf die Zahl der Transkriptausschnitte der jeweiligen Gruppendiskussion, in denen soziale Kategorisierungen von Schülerinnen und Schülern nach der inhaltlichen Kodierung eine Rolle spielen (vgl. Vogler et al. 2018). Damit erhält man ein grobes Maß für die Grundmenge in unserem Sub-Korpus.

Der Vergleich auf der Basis des Diagramms in Abb. 3 zeigt bereits auf den ersten Blick deutliche Unterschiede zwischen den Lehrkräften verschiedener Fächer: Die Mathematiklehrkräfte beider Schulformen (GD 3) nutzen z. B. viele verschiedene Kollektionen, während die Deutschlehrkräfte (GD 4) überwiegend nach INSTITUTIONELLER ROLLE kategorisieren und ansonsten - bei ähnlicher Häufigkeit - nur drei weitere Kollektionen nutzen.

Die Betrachtung der Verteilungen zwischen den Kollektionen LEBENSPHASE und INSTITUTIONELLE ROLLE weist - wie oben dargestellt - implizit darauf hin, ob die Schülerinnen und Schüler in ihrer institutionellen Rolle, z. B. als ,Fünftklässler‘, oder auch außerschulisch als ,Kinder‘ sozial relevantgesetzt werden. Dass die Gruppe der Deutschlehrkräfte beider Schulformen (bei insgesamt wenigen NSK) sehr viel häufiger entlang der Kollektion InSTITUTIONELLE ROLLE kategorisiert als die anderen Gruppen, ist besonders interessant unter dem Gesichtspunkt, dass gerade in dieser Gruppe die Kollektion LEISTUNGSVERMÖGEN (,die Fitten', ,die Schwachen`) gar nicht vorkommt. Die Deutsch-Lehrkräfte kategorisieren institutionell also nach dem Niveau der Schullaufbahn (,Fünftklässler` vs. ,Oberstufenschüler'), halten aber Leistungsunterschiede innerhalb der Jahrgangsstufen im Umgang mit den präsentierten Videoausschnitten offenbar für weniger relevant. In der Gruppendiskussion 1 der Gymnasiallehrkräfte beider Fachrichtungen wird dagegen die Kollektion LEISTUNGSVERMÖGEN proportional am häufigsten angesprochen. Bemerkenswert ist allerdings generell, dass Kategorisierungen nach LEISTUNGSVERMÖGEN, wiewohl sie zum diagnostischen und bewertenden Kern unterrichtlicher Tätigkeit gehören und auch durchaus vorkommen, im Vergleich zu den anderen Kollektionen durchgängig eine eher geringe Rolle spielen.

Hinsichtlich der Kontrastierung der Schulformen fällt weiterhin auf, dass in der Gruppendiskussion 1 der am Gymnasium Unterrichtenden beider Fächer so gut wie gar nicht auf das SOZIALE MILIEU verwiesen wird, während dies bei den Gesamtschullehrkräften (L-GD 2) sehr viel häufiger der Fall ist. Auch in den übrigen Gruppendiskussionen 3 und 4 wird dieser Aspekt, wenn er vorkommt, vornehmlich von den Gesamtschullehrkräften thematisiert (Ausnahme: eine Gymnasiallehrerin, deren Schule sich durch einen hohen Anteil von Jugendlichen mit Migrationshintergrund auszeichnet). Für Gymnasiallehrkräfte scheint also beim professionellen Blick auf die in den Videosequenzen 
agierenden Schülerinnen und Schüler SOzIALES MILIEU i.d.R. kein relevantes soziales Ordnungsprinzip zu sein. Die Relevanz einer entsprechenden Bedeutsamkeit rückt erst durch ein ,auffälliges‘, d. h. hier eher benachteiligtes, soziales Umfeld der Schule in den Blick. Unter dem Aspekt der zunehmenden Heterogenität auch der Schülerschaft des Gymnasiums ist dies ein bemerkenswertes Ergebnis.

Im Zusammenhang mit dem SOZIALEN MILIEU fällt darüber hinaus auf, dass die Mehrsprachigkeit von Lernenden in den Betrachtungen der Lehrenden nur auf Nachfrage thematisiert wurde, obwohl unsere Videoclips Schülerinnen und Schüler mit familialer Migrationsgeschichte enthielten.

Ebenfalls interessant ist im Kontext dieser Überlegungen, dass auch die Kollektion GENDER, obwohl in pädagogischen Diskursen gegenwärtig immer noch hoch relevant, in allen Gruppendiskussionen der Lehrenden kaum eine Rolle spielt: Wenn Lehrer Schülerverhalten kommentieren, unterscheiden sie sprachlich nicht nach ,Mädchen“ und ,Jungen‘. Ebenso deutlich fällt dieser Befund bei den Kollektionen PSYCHOLOGISCHE KATEGORIEN (,cooler Typ', ,ein Schüchterner') und FAmiliE (z. B. ,Bruder', ,Schwester`) aus, auf die in den LehrendenGruppendiskussionen nur ganz vereinzelt zurückgegriffen wird.

Jede Art von Heterogenität, sei sie der sozialen Herkunft, der Geschlechtszugehörigkeit, der Persönlichkeit und bedingt sogar der Leistungsfähigkeit geschuldet, scheint also in der Perspektive der Lehrenden hinter der institutionellen Rolle des Kollektivs der Klasse eher verdeckt zu sein. Aus den abgestuften Häufigkeiten der Kollektionen InSTITUTIONELLE ROLLE, LEBENSPHASE und - mit deutlichem Abstand - LeistungSvermöGEN und soziales Milieu (s. Abb. 3 oben) kann man schließen, dass Lehrende ihre Klassen als kollektive Adressaten (Heller 2017) ansehen und dabei allenfalls Leistungs- und - selten und nur unter bestimmten Bedingungen - soziale Unterschiede für relevant halten, nicht aber Geschlechtsunterschiede oder persönliche Merkmale einzelner Schülerinnen und Schüler.

Durch die Kontrastierung der NSK zwischen verschiedenen Diskussionsgruppen der Lehrenden erhalten wir somit einen Hinweis darauf, dass sich die implizite professionelle Sicht auf die soziale Welt des Klassenzimmers seitens der Lehrkräfte unterscheidet (vgl. auch neuere Studien zur Mikrokultur im Klassenzimmer verschiedener Lehrender: Erath 2017), und zwar besonders nach Schulform, aber auch nach Fachkultur und sozialer Zusammensetzung der Schülerschaft. Vor diesem Hintergrund wendet sich der folgende Abschnitt den entsprechenden Häufigkeitsverteilungen aus den Gruppendiskussionen der Schülerinnen und Schüler zu. 


\subsection{Die impliziten Relevantsetzungen in NSK von Schülerinnen und Schülern}

Die Analyse der Gruppendiskussionen von Schülerinnen und Schülern ergab, dass hier zwar im Wesentlichen auf dieselben Kollektionen wie auch in den Lehrer-Diskussionen zurückgegriffen wurde, dass jedoch ein membership categorization device zusätzlich auftauchte, das bei den Lehrkräften nicht vorkam: ROLle IN DER Klasse als PeERGRouP (,Außenseiter', ,Beliebter‘, ,Streber‘, ,Schlaumeier'). Die Liste der implizit relevant gesetzten Kollektionen umfasst mithin insgesamt die folgenden Kategorisierungsverfahren:

- LEBENSPHASE (,Kinder')

- Institutionelle Rollen (,Schüler‘, ,Fünftklässler`)

- LeISTUngSvermögen (,die Fitten', ,die Dummen', ,die Schwachen‘ etc.)

- Soziales Milieu (,Kinder mit Migrationshintergrund‘, ,Ausländer‘, ,Kinder aus verschiedenen Grundschulen/Förderschulen').

- Psychologische Kategorien (,cooler Typ')

- Familie (zur Bezugnahme auf das private Umfeld: ,Vater', ,Mutter‘, ,Bruder`)

- Rolle IN DeR KLASSE Als PeERGRoup (,Außenseiter', ,Beliebter', ,Streber‘, ,einer, der nicht immer aufpasst').

Wir berichten hier zunächst die Häufigkeitsverteilungen und präsentieren in diesem Zusammenhang vor dem Hintergrund der Analysen in 5.2 einige Beispiele, die für die Verwendung in den Schüler-Gruppendiskussionen typisch sind.

Abbildung 4 zeigt die NSK über die einzelnen Diskussionsgruppen hinweg in absoluten Zahlen, bezogen auf die jeweilige Menge der einschlägigen Transkriptausschnitte. Wichtig ist dabei der Hinweis, dass sich in den Schüler-Diskussionen insgesamt deutlich weniger NSK als in den Diskussionen der Lehrenden finden. Das liegt daran, dass hier häufiger unspezifische Referenzformen (man, manche, jeder, niemand) oder Pronomen wie wir oder der verwendet werden. Die methodischen Vorbehalte gegenüber einer Generalisierung unserer Beobachtungen (vgl. Abschnitt 5.2 oben) haben hier also auf Grund der niedrigeren Zahlen besonderes Gewicht.

Hier wird bspw. sichtbar, dass eine Gruppe privilegierter Gymnasiasten (2H-J-L2) die institutionenbezogene Kollektion im Vergleich zu anderen Kollektionen häufiger benutzt. Beim Rückgriff auf die Kollektion der INSTiTUTioNELLEN ROLLE wird insgesamt sehr viel seltener als bei den Lehrenden auf die 


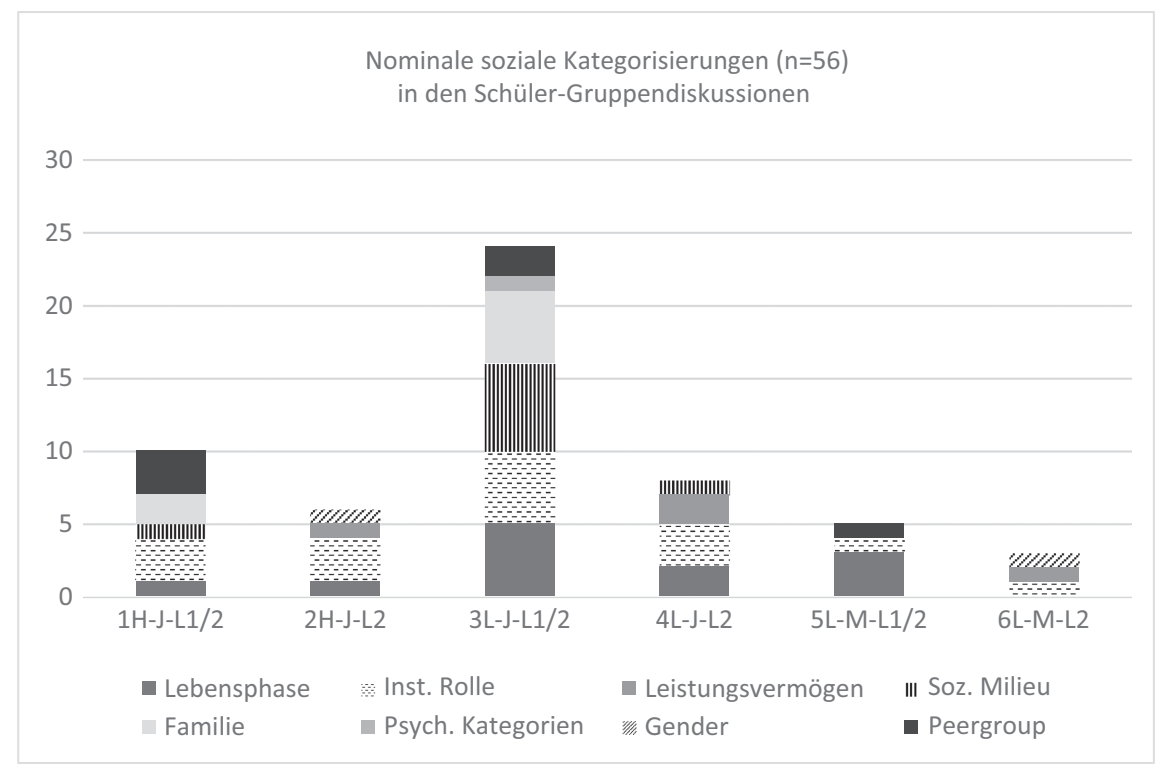

Abb. 4: Kollektionen in den Schüler-Gruppendiskussionen.

pauschalisierende Kategorie ,Schüler' zurückgegriffen. Eher wird kategorial nach Klassenstufen, Schulformen oder sogar Bundesländern unterschieden:

Beispiel (31) 1H-J-L1/2 (Jungen Gym, L1/L2)

1515 ole das kommt mir so vor als wären die erst in der VIERten klasse.

Beispiel (32) 2H-J-L2 (Jungen Gym, L2) (im Kontrast zu „Förderschulen“):

442 zek bei_nem gymNAsium ist das ja was anderes.

443 dann MÜSsen die das ja wissen.

Beispiel (33) 2H-J-L2 (Jungen Gym, L2)

1541 ulf die sind irgendwie WEIter.

1542 zek ja die sind auch in einem anderen LAND.

1543 da MUSS man auch andere ( ).

1544 ulf BuNdesland.

1545 mar in_nem anderen BUNdesland. 
Dagegen wird in der Mädchen-Gruppe (5L-M-L1/2, Gesamtschülerinnen) bei einem insgesamt niedrigen Vorkommen vornehmlich nach der LEBENSPHASE kategorisiert - eine Verteilung, die bei den Lehrkräften nie vorkommt. Ansonsten wird die Referenzform auch bei den Lernenden recht explizit auf die LEBENSPHASE und die außerschulischen Bedingungen der 11- bis 12jährigen Kinder bezogen:

Beispiel (34) 1H-J-L1/2 (Jungen Gym, L1/L2)

0456 gle sonst denkt der was ist denn DAS für ein unerzogenes kind?

LEISTUNGSVERMÖGEN als categorization device spielt bei den Schüler-Diskussionen kaum eine Rolle. Nur einmal werden Schülerinnen und Schüler in den Klassen pauschal nach ihrem LEISTUNGSVERMÖGEN differenziert:

Beispiel (35) 6L-M-L2 (Mädchen, Ges, L2)

1823 til es gibt ja manche SCHLAU (.) sehr schlaue in der klasse.

Ansonsten wird Leistungsfähigkeit im Zusammenhang mit der Prozessierung von Informationen kategorisiert, wodurch sich Verbindungen zur thematischen Relevanz der Verständlichkeit von Schüler-Erklärungen (4.2.3.5 oben) ergeben:

Beispiel (36) 2H-J-L2 (Jungen Gym, L2)

1011 mar und die andern kinder also.

1012 oder manche die EH schon probleme damit haben?

Beispiel (37) 4L-J-L2 (Jungen Ges, L2)

0603 wal also wer das nicht verSTEHT, 0604 kann RUNden nicht kapieren.

Wir stellen also fest, dass wir in den Schüler-Diskussionen starke Unterschiede im Blick auf Unterricht als institutionelles Geschehen finden, die die soziale Herkunft der Diskutierenden widerspiegeln.

Das SOZIALE MiLIEU wird nur in einer Gruppe (3L-J-L1/2) von ein- und mehrsprachigen Jungen aus einer Gesamtschule mit eher niedrigem Sozialstatus nennenswert zur Kategorisierung genutzt. Bemerkenswert ist in diesem Zusammenhang eine Gemeinsamkeit zwischen Schüler- und Lehrer-Gruppendiskussio- 
nen: Eine NSK nach ethnischer Zugehörigkeit o. Ä. wird trotz entsprechendem augenfälligen Status einiger Schüler in den Videos auch von den Lernenden nur auf Nachfragen hin vorgenommen. Ohne Steuerung auf diese Unterscheidung tauchte eine derartige Einordnung nur einmal in einer Gruppe von zweisprachigen Gesamtschülern auf, und hier auch nicht als NSK, sondern als zugeschriebene Eigenschaft:

Beispiel (38) 4L-J-L2 (Jungen Ges, L2)

0226 dar ich glaub der Kostas kommt aus einem anderen LAND und ähm-

Im Unterschied zu dem beschriebenen Formulierungsaufwand, den Lehrpersonen bei der Bezugnahme nach der Kollektion ETHNISCHE ZUGEHÖRIGKEITEN betreiben (s. o. Abschnitt 5.2), haben Schülerinnen und Schüler einer Gruppe von L1- und L2-Gesamtschülern aber offenbar keine Scheu, Personen nach ihrer nationalen oder ethnischen Herkunft zu benennen, wenn dies zum Thema gemacht wird:

Beispiel (39) 3L-J-L1/2 (Jungen Ges, L1/L2)

1109 nas also ICH merk das direkt wenn ein türke

1110 deutsch redet.

Dabei werden sogar die sprachlichen Positionierungsprozesse registriert:

Beispiel (40) 3L-J-L1/2 (Jungen Ges, L1/L2)

1100 nas ( ) mal ist der so türke?

1101 mal redet der so äh ja ich äh.

1102 zum beispiel so komisch äh.

PSYCHOLOGISCHE KATEGORIEN zur sozialen und genderbezogenen Einordnung der Lernenden sowie GENDER sind auch in den Gruppendiskussionen der Lernenden nur selten zu finden. Die Kollektion GENDER ist dabei insofern bemerkenswert, als sie typischerweise in Form der NSK ,Mädchen“, nicht etwa ,Junge“ erscheint. Die soziale Ordnung nach Geschlecht scheint also eher dann bedeutsam zu werden, wenn es um Mädchen geht, woraus man vorsichtig schließen kann, dass Jungen in der sozialen Wahrnehmung durch die Fünftklässler eher den „Normalfall“ darstellen, den man nicht sprachlich herausheben muss. Die Kol- 
lektion FAMILIE erhält im thematischen Zusammenhang der Erörterung der häuslichen Sprachverwendung in Gruppe 3 einen gewissen inhaltlichen Stellenwert.

Die Kategorisierung nach der ROLLE IN DER KLASSE ALS PEER GROUP in einzelnen Gruppen ist besonders unter dem Gesichtspunkt bedeutsam, dass eine entsprechende Kategorisierung bei den Lehrkräften nie vorkommt. In der Gymnasiastengruppe (1H-J-L1/2) wird bspw. in Bezug auf einen Schüler im Video spekuliert, dass er „nicht so ein beLIEBter ist“ (Z. 213) bzw. „halt so_n außenseiter“ (Z. 223) sei, in Gruppe 3L-J-L1/2 geht es um „so einen der NICHT so immer aufpasst“ (Z. 360), wobei das erste „so“ jeweils die Typisierung indiziert.

Es gibt also einige Passungen der Sichtweisen von Schülerinnen und Schülern zu denen der Lehrpersonen, aber eher bei privilegierten Schüler-Gruppen. Ansonsten finden wir vor allem Divergenzen: Pauschalisierende Einordnungen einfach als ,Schüler' sind seltener, LEISTUNGSVERMÖGEN steht eher im Zusammenhang mit dem konkreten Verstehen des Unterrichtsstoffes und weniger mit pauschalen Zuschreibungen wie ,Schlaue‘. Die Kollektion ROLLEN IN DER KLASSE ALS PEERGROUP wird ausschließlich von den Schülerinnen und Schülern verwendet.

Nach dieser Bestandsaufnahme der sozialen Kategorisierungen bei den Lernenden widmen wir den folgenden Abschnitt dem vergleichenden Blick auf die Perspektivierung von Unterricht durch Lehrende und Lernende.

\subsection{Passungen und Divergenzen in der Perspektivierung der sozialen Einheit Schulklasse}

Die folgenden Aussagen zum Vergleich der aus den NSK ableitbaren Vorstellungen zur sozialen Ordnung im Klassenzimmer gelten angesichts der Innovativität des methodischen Ansatzes zunächst nur für unsere Daten. Unter dem Gesichtspunkt, dass sämtliche Stimuli und äußeren Bedingungen bei allen Gruppendiskussionen konstant gehalten wurden, sind aus den Vergleichen jedoch interessante Hinweise auf das analytische Potential der vorgestellten Analyseform abzuleiten.

Bereits die größere Differenziertheit in den genutzten Kollektionen durch Schülerinnen und Schüler im Vergleich zu Lehrkräften kann als erster Hinweis darauf betrachtet werden, dass die Vorstellungen von Lehrpersonen zur Mikrokultur von Unterricht stark von ihrer - z. T. Fächer und Schulformen übergreifenden - Professionalität geprägt sind. Lernende hingegen scheinen in der Verarbeitung der Situation nach dem Wechsel in eine neue Schulform stärker durch ihr persönliches Erleben gesteuert. Dieses Erleben ist weniger durch eine 
verbindende professionelle Identität als Fünftklässler gefiltert, sondern nach familialer Herkunft und bisherigen Schulerfahrungen sehr unterschiedlich. Die im Vergleich geringere Rolle und die andere Qualität, die die INSTITUTIONELLE ROLLE bei den Bezeichnungen in den Schüler-Diskussionen spielt, legt nahe, dass die schulische Rollenzuschreibung ,Schüler' als Gegenpart zum ,Lehrer“ für die meisten Lernenden eben nicht - wie bei den Lehrenden - vorherrschend ist. Das gilt hier, obwohl im Gesamtkorpus aller Gruppendiskussionen von Lernenden auch häufig pauschalisierend von ,Klasse“ die Rede ist. In diesem Zusammenhang ist interessant, dass die Diskussionsgruppen von Gymnasiasten mit einem besonders privilegierten sozialen Hintergrund, die auch inhaltlich das professionelle didaktische Handeln der beobachteten Lehrpersonen auffällig oft zum Thema machen (vgl. Vogler et al. 2018), auch den höchsten Anteil an NSK der InSTITUTIONELLEN ROLLE aufweisen. Demgegenüber spielt diese Perspektive in anderen Diskussionsrunden (bspw. von Mädchen aus eher benachteiligten sozialen Milieus) fast keine Rolle.

Besonders interessant ist, dass die Lernenden auf eine Kollektion zurückgreifen, die bei den Lehrenden gar nicht vorkommt: Die Relevantsetzung der RoLLE IN DER KLASSE ALS PEERGROUP ist der stärkste Indikator dafür, dass Schülerinnen und Schüler den Klassenverband als eine differenzierte soziale Formation sehen, die auch durch gruppendynamische Rollen- und Statusprozesse geprägt ist.

Passungen und Divergenzen in den Sichtweisen von Lehrenden und Lernenden auf die soziale Formation in Klassenzimmern können in der folgenden Weise zusammengefasst werden:

- Die Reduzierung der Lernenden auf ihre institutionelle Rolle als Schüler (der fünften Klasse) ist bei den Lehrenden deutlich durchgängiger als bei den Lernenden.

- Lernende nehmen ihre Peers demgegenüber stärker in unterschiedlichen sozialen Zugehörigkeiten wahr, wozu auch die Rolle in der Klassengemeinschaft gehört.

- Die Einordnung nach Leistungsvermögen ist auch bei den Lehrkräften weniger durchgängig als erwartbar, spielt aber bei den Lernenden kaum eine Rolle.

- Das soziale Milieu der Heranwachsenden ist weder in der Sichtweise von Lehrenden noch der von Lernenden durchgängig prominent, die Unterscheidung L1-/L2-Sprecher wird weder von Lehrenden noch von Lernenden relevant gesetzt.

- Die Kategorisierung nach persönlichen Merkmalen (wie etwa schüchtern) spielt weder bei Lehrpersonen noch bei Schülerinnen und Schülern eine große Rolle. 
Aus der Sicht der Lehrenden erscheint die Klasse also eher als Kollektiv, als komplementärer Part zur Lehrperson im unterrichtlichen Geschehen, während die Schülerinnen und Schüler ihre Mitschüler deutlich differenzierter und auch z. T. nach anderen Ordnungsprinzipien sozial kategorisieren. Die Klassengemeinschaft wird hier zwar ebenfalls als Gruppe gesehen, weswegen persönliche Merkmale Einzelner wie Herkunft, Geschlecht oder Charakter i.d. R. weniger relevant sind, aber eher als soziale Gruppe denn als Lerngemeinschaft.

Prägnant formuliert: Unterricht ist für Lehrende ein Raum professionellen Handelns, in dem Schülerinnen und Schüler als Kollektiv Ziel und Objekt der Vermittlung sind. Für Schülerinnen und Schüler ist Unterricht ein vielfältig differenzierter sozialer Raum, der je nach sozialer Herkunft anders perspektiviert wird.

Grundlegend scheint es also eher Divergenzen als Passungen in den impliziten Relevantsetzungen der Beteiligten im Klassenzimmer zu geben. Gerade die Implizitheit dieser Sichtweisen, die durch unser methodisches Vorgehen aufgedeckt wurden, aber den Beteiligten selbst nicht zugänglich sein dürften, macht die Beobachtungen unter Anwendungsaspekten wertvoll. Viele der an den Gruppendiskussionen beteiligten Lehrpersonen würden möglicherweise im professionellen Diskurs auf Nachfrage die Heterogenität im Klassenzimmer und die Notwendigkeit individueller Förderung betonen, obwohl sie die Schülerinnen und Schüler gemäß ihren Kategorisierungen eher als Lernkollektiv auffassen. Die Sensibilisierung für solche pauschalisierenden Kategorisierungen der Lernenden dürfte ein wesentlicher Schritt zur Veränderung von Routinen sein. Die Einsicht in die Diversität nicht nur der Lernvoraussetzungen, sondern auch des Erlebens von Unterrichtsalltag als sozialem Geschehen dürfte Lehrende dazu in die Lage versetzen, Partizipationsgelegenheiten adäquater zuzuschneiden. Lernende wiederum dürften in dem Maße am Unterrichtsgeschehen partizipieren, in dem ihre Sicht auf die soziale Gemeinschaft im Klassenzimmer der der Lehrenden ähnlich ist.

Im Folgenden führen wir die Ergebnisse der beiden analytischen Zugänge unter dem Gesichtspunkt der Förderung von Partizipationschancen von Schülerinnen und Schülern zusammen.

\section{Zusammenführung der analytischen Zugänge}

Sowohl die Analyse der thematischen Bezugnahmen auf Schüleräußerungen (Abschnitt 4) als auch die Rekonstruktion der impliziten Sichtweisen auf die soziale Ordnung im Klassenzimmer über die sozialen Kategorisierungen (Abschnitt 5) haben systematische Passungen und Divergenzen innerhalb der jeweiligen 
Gruppen sowie zwischen Lehrpersonen einerseits und Schülerinnen und Schülern andererseits ergeben. Während die Ähnlichkeiten und Übereinstimmungen ein Schlaglicht auf die institutionelle Lehr-/Lernkultur in unseren Klassenzimmern einschließlich ihrer ,blinden Flecken' werfen können, eröffnen die aufgedeckten Divergenzen in den Sichtweisen einen Zugang zu möglicherweise nicht eingelösten wechselseitigen Erwartungen.

$\mathrm{Zu}$ den interessanten Passungen zwischen Lehrenden und Lernenden gehören in beiden methodischen Zugängen gerade die Bereiche, die von den Diskutierenden weder thematisch noch implizit in einem wesentlichen Ausmaß relevant gesetzt werden. Hier sind wichtige Aspekte der Sprachlichkeit von Schüleräußerungen (4.2.3) ebenso $\mathrm{zu}$ nennen wie das weitgehende Ignorieren der sozialen und ethnischen Herkunft von Schülerinnen und Schülern (5.3 und 5.4), die ja besondere Anforderungen an die Sprachsensibilität des Unterrichtens stellen kann. Obwohl der Zusammenhang von sprachlichem und fachlichem Lernen (z. B. Becker-Mrotzek et al. 2013) sowie die Unterrichtsinteraktion als Lernumgebung (Heller \& Morek 2015; Morek \& Heller in diesem Band) mehr und mehr in den Blick der Forschung geraten, dürften partizipationsförderliche Aktivitäten auch in Zeiten von Inklusion schon deshalb nicht umgesetzt werden, weil sich die Sprachlichkeit des Unterrichtsprozesses und das Ausmaß der sprachbedingten verschiedenen schülerseitigen Partizipationsmöglichkeiten gar nicht im Aufmerksamkeitsraum von Lehrenden und Lernenden befinden.

Die Befunde in beiden Zugängen zur Verschiedenheit der Sichtweisen auf denselben Unterricht jeweils innerhalb der Gruppe der Lehrenden und der Lernenden sowie zwischen beiden Gruppen lassen sich unterschiedlich deuten: Die Divergenzen zwischen den Lehrpersonen sind offensichtlich mit unterschiedlichen professionellen Konzepten und Erfahrungen zu erklären. Sie zeigen, dass eine sozial und kognitiv heterogene Schülerschaft mindestens bei Gymnasiallehrkräften auch heute noch als eine Art ,abweichender Fall vor dem Hintergrund der Annahme einer mit den Jahrgangsstufen gegebenen Leistungshomogenität gesehen wird (5.2). Die Unterschiede zwischen den Sichtweisen der Lernenden sind demgegenüber eher sozial bedingt. Heterogenitäten unter den Schülerinnen und Schülern zeigen sich hier besonders deutlich in den Relevantsetzungen der institutionellen Aufgabenverteilung und Rollenzuweisungen. Hier legen beide Zugänge nahe, dass privilegierte Fünftklässler eher die professionelle Bedingtheit des Unterrichtsgeschehens und die Verantwortung der Lehrenden für die Partizipationschancen der Lernenden betonen: Sie sehen z. B. die Verständlichkeit von Erklärungen als Aufgabe der Lehrpersonen, während weniger privilegierte Schülerinnen und Schüler auch von ihren Mitlernenden verlangen, dass sie ihre Erklärungen auf die Verarbeitungsmöglichkeiten ihrer Peers zuschneiden (4.2.3.5). Die größere Passung 
zur Sichtweise der Lehrenden bei privilegierten Schülerinnen und Schülern zeigt sich auch in der impliziten Kategorisierung der Lernenden nach ihrer INSTITUTIONELLEN RoLLE, die sich häufiger bei den Gymnasiasten als bei benachteiligten Diskutierenden findet (5.3.).

Besonders aussagekräftig sind die aufgedeckten Divergenzen in den Sichtweisen zwischen Lehrenden und Lernenden. Beide analytischen Zugänge arbeiten jeweils schülerspezifische Aspekte der jeweiligen Deutungsmuster heraus, die bei den Lehrenden gar nicht vorkommen: In ihren thematischen Relevantsetzungen erwähnen Lehrende nie die o.a. Erwartung, dass Schülererklärungen und -begründungen auch für die Mitlernenden verständlich sein sollten. Eine Diskrepanz zwischen dem Verstehen und dem Erklärenkönnen wird ebenfalls nur von den Lernenden angemerkt (4.2.3.1), genauso wie nur sie ihre Peers implizit nach ihrer sozialen Rolle in der Klassengemeinschaft kategorisieren (5.3). So unterschiedlich diese Aspekte sein mögen, die jeweils exklusiv von Schülerinnen und Schülern relevant gesetzt werden: Sie zeigen, dass es offensichtlich sowohl didaktische als auch soziale Aspekte des Unterrichtsgeschehens gibt, die für Lernende eine Rolle spielen, die aber von Lehrpersonen ausgeblendet werden.

Im Hinblick auf die Nutzbarkeit unserer Ergebnisse für die einleitend angesprochene Qualifizierung von Lehrpersonen muss also zunächst einmal ein Bewusstsein darüber vermittelt werden, in welcher Weise wechselseitige Erwartungen zwischen Lehrenden und Lernenden bezüglich des Unterrichtsgeschehens nicht kongruent sind. In dem Maße, in dem die Herstellung von Passungen Aufgabe der Lehrperson ist, muss letztere qualifiziert werden, die Mechanismen von Passungen und Divergenzen zu durchschauen und professionell mit ihnen umzugehen. Dabei ist es entscheidend, die unterschiedlichen Nähe- und Distanzverhältnisse zwischen sozial mehr oder weniger privilegierten Schülerinnen und Schülern zu erkennen.

Die Entdeckungen der vorliegenden Analyse werden gestützt durch die weiteren Ergebnisse der breiter angelegten Gruppendiskussionsstudie von InterPass, die stark zusammenfassend ergab, dass Lehrende primär auf das eigene professionelle Handeln und weniger auf die schülerseitigen Angebote, Lerngelegenheiten oder Unterschiedlichkeiten in den Ressourcen orientiert sind (Vogler et al. 2018). Rekonstruktionen von unterrichtlichen Sequenzen, die z. B. das mangelnde Aufgreifen schülerseitiger Beiträge dokumentieren (vgl. Prediger et al. 2016; Heller 2017), können in der Tat durch den hier vorgestellten zusätzlichen methodischen Zugang triangulativ ergänzt (vgl. auch WagnerWilli 2010) und durch Auffassungen zur professionellen Rolle erklärt werden (s. o. Abschnitt 1).

Unsere Beobachtungen müssen sicher durch weitere Daten und Analysen auf breitere empirische Grundlagen gestellt werden. Insgesamt hat sich jedoch 
aus unserer Sicht die Fruchtbarkeit der beiden methodischen Zugänge erwiesen, und zwar sowohl in methodischer Hinsicht als auch bezogen auf die empirischen Erträge. Mit Blick auf anwendungsbezogene Fragestellungen vermochten beide der hier vorgestellten methodischen Zugänge z. T. unausgesprochene Überzeugungen und Orientierungen von Lehrpersonen sichtbar zu machen, an denen Fortbildungskonzepte ansetzen müssen, die das Ziel verfolgen, partizipationsund diskurserwerbsförderliche Unterstützungsverfahren zu vermitteln. Sie ermöglichen es zudem, Lehrpersonen Anforderungen und Wirkungen ihres Handelns auf Lernende zugänglich zu machen, die ihnen in der Komplexität und Flüchtigkeit des Unterrichtsgeschehens verborgen bleiben. Davon dürften langfristig Lehrende und Lernende profitieren.

\section{Literatur}

Auer, Peter (1995): Ethnographic Methods in the Analysis of Oral Communication. Some Suggestions for Linguists. In Uta Quasthoff (Hrsg.), Aspects of oral communication, 419440. Berlin: De Gruyter.

Baumert, Jürgen \& Kunter, Mareike (2006): Stichwort: Professionelle Kompetenz von Lehrkräften. Zeitschrift für Erziehungswissenschaft 9 (4), 469-520.

Baumert, Jürgen, Mareike Kunter, Martin Brunner, Stefan Krauss, Werner Blum \& Michael Neubrand (2004): Mathematikunterricht aus Sicht der PISA-Schülerinnen und -Schüler und ihrer Lehrkräfte. In Manfred Prenzel, Jürgen Baumert, Werner Blum, Rainer Lehmann, Detlev Leutner \& Michael Neubrand (Hrsg.), PISA 2003. Der Bildungsstand der Jugendlichen in Deutschland - Ergebnisse des zweiten internationalen Vergleichs, 314-354. Münster: Waxmann.

Becker-Mrotzek, Michael, Karen Schramm, Eike Thürmann \& Johannes Vollmer (Hrsg.) (2013), Sprache im Fach - Sprachlichkeit und fachliches Lernen. Münster: Waxmann.

Bohnsack, Ralf (2010): Rekonstruktive Sozialforschung. Einführung in qualitative Methoden. 8. Aufl. Opladen \& Farmington Hills: Budrich.

Bromme, Rainer (1992): Der Lehrer als Experte. Zur Psychologie des professionellen Wissens. Münster: Waxmann.

Bühler, Karl (1999/1934): Sprachtheorie. Die Darstellungsfunktion der Sprache. Stuttgart: Lucius \& Lucius.

Buttlar, Ann-Christin (2019): Sequenzielle Analysen interaktiver Verfahren des lehrerseitigen Umgangs mit Schüleräußerungen. In Katharina Verrière \& Larena Schäfer (Hrsg.), Interaktion im Klassenzimmer. Forschungsgeleitete Einblicke in das Geschehen im Unterricht, 97-117. Wiesbaden: Springer VS.

Cedersund, Elisabet \& Lennart Svensson (1996): A ‘Good' or a 'Bad' Student: A Study of Communication in Class Assessment Meetings. Language and Education 10 (2-3), 132-150.

Cekaite, Asta (2012): Affective Stances in Teacher-Novice Student Interactions. Language, Embodiment, and Willingness to Learn in a Swedish Primary Classroom. Language in Society 41 (5), 641-670. 
Claussen, Marten (2002): Unterrichtsqualität. Eine Frage der Perspektive? Empirische Analysen zur Übereinstimmung, Konstrukt- und Kriteriumsvalidität. Münster: Waxmann.

Ditton, Hartmut (2002): Lehrkräfte und Unterricht aus Schülersicht. Ergebnisse einer Untersuchung im Fach Mathematik. Zeitschrift für Pädagogik 48 (2), 262-286.

Erath, Kirstin (2017): Mathematisch diskursive Praktiken des Erklärens. Wiesbaden: Springer. Hausendorf, Heiko (2000): Zugehörigkeit durch Sprache. Eine linguistische Studie am Beispiel der deutschen Wiedervereinigung. Tübingen: Niemeyer.

Heller, Vivien (2012): Kommunikative Erfahrungen von Kindern in Familie und Unterricht. Passungen und Divergenzen. Tübingen: Stauffenburg.

Heller, Vivien (2017): Managing knowledge claims in classroom discourse. The public construction of a homogeneous epistemic status. Classroom Discourse 8 (2), 156-174.

Heller, Vivien \& Morek, Miriam (2015): Unterrichtsgespräche als Erwerbskontext: Kommunikative Gelegenheiten für bildungssprachliche Praktiken erkennen und nutzen. leseforum.ch 3, 1-23.

Heller, Vivien \& Uta Quasthoff (2020): Lehrerhandeln aus Schülersicht: Gruppendiskussionen sozial privilegierter und benachteiligter Lernender. In Helga Kotthoff \& Vivien Heller (Hrsg.), Ethnografien und Interaktionsanalysen im schulischen Feld. Diskursive Praktiken und Passungen interdisziplinär, 89-115. Tübingen: Narr Francke Attempto Verlag.

Heller, Vivien, Uta Quasthoff, Susanne Prediger \& Anna Vogler (2017): Bildungssprachliche Praktiken aus professioneller Sicht: Wie deuten Lehrende Schülererklärungen und -begründungen? In Bernt Ahrenholz, Britta Hövelbrinks \& Claudia Schmellentin (Hrsg.), Fachunterricht und Sprache in schulischen Lehr-/Lernprozessen, 139-160. Tübingen: Narr.

Hofer, Manfred (1981): Schülergruppierungen in Urteil und Verhalten des Lehrers. In Manfred Hofer (Hrsg.), Informationsverarbeitung und Entscheidungsverhalten von Lehrern. Beiträge zu einer Handlungstheorie des Unterrichtens, 192-221. München: Beltz.

Holodynski, Manfred, Mirjam Steffensky, Bernadette Gold, Christina Hellermann, Cornelia Sunder \& Anja Fiebranz (2017): Lernrelevante Situationen im Unterricht beschreiben und interpretieren. Videobasierte Erfassung professioneller Wahrnehmung von Klassenführung und Lernunterstützung im naturwissenschaftlichen Grundschulunterricht. In Cornelia Gräsel \& Kati Trempler (Hrsg.), Entwicklung von Professionalität pädagogischen Personals, 283-302. Wiesbaden: Springer.

Klieme, Eckhard, Nina Jude, Dominique Rauch, Holger Ehlers, Andreas Helmke \& Wolfgang Eichler (2008): Alltagspraxis, Qualität und Wirksamkeit des Deutschunterrichts. In Eckhard Klieme (Hrsg.), Unterricht und Kompetenzerwerb in Deutsch und Englisch. Ergebnisse der DESI-Studie, 319-344. Weinheim: Beltz.

König, Katharina (2014): Spracheinstellungen und Identitätskonstruktion. Eine gesprächsanalytische Untersuchung sprachbiographischer Interviews mit DeutschVietnamesen. Berlin: De Gruyter.

Koole, Tom \& Ed Elbers (2014): Responsiveness in teacher explanations: A conversation analytical perspective on scaffolding. Linguistics and Education 26, 57-69.

Kotthoff, Helga (2012): Lehrer(inne)n und Eltern in Sprechstunden an Grund- und Förderschulen - Zur interaktionalen Soziolinguistik eines institutionellen Gesprächstyps. Gesprächsforschung - Online-Zeitschrift für verbalen Interaktion (13), 290-321.

Kotthoff, Helga (2014): Faul wie e Hund. Kritische Eltern in der schulischen Sprechstunde. Freiburger Arbeitspapiere zur germanstischen Linguistik 22, 1-12. 
Leßmann, Ann-Christin (i. E.): Unterrichtsinteraktion in der Grundschule. Sequenzielle Analysen zur Ko-Konstruktion von Angemessenheit zwischen Lehrenden und Lernenden. Tübingen: Stauffenburg.

Luckmann, Thomas (1986): Grundformen der gesellschaftlichen Vermittlung des Wissens: Kommunikative Gattungen. Kölner Zeitschrift für Soziologie und Sozialpsychologie (Sonderheft 27), 191-211.

Luhmann, Niklas (1997): Die Kunst der Gesellschaft. 5. Aufl. Frankfurt a.M.: Suhrkamp. Mannheim, Karl (1980/1924): Eine soziologische Theorie der Kultur und ihrer Erkennbarkeit. In David Kettler, Volker Meja \& Niko Stehr (Hrsg.), Karl Mannheim. Strukturen des Denkens, 155-322. Frankfurt a.M.: Suhrkamp.

Meuser, Michael \& Reinhold Sackmann (1992). Zur Einführung: Deutungsmusteransatz und empirische Wissenssoziologie. In Michael Meuser \& Reinhold Sackmann (Hrsg.), Analyse sozialer Deutungsmuster. Beiträge zur empirischen Wissenssoziologie, 9-38. Pfaffenweiler: Centaurus.

Margutti, Piera \& Paul Drew (2014): Positive evaluation of student answers in classroom instruction. Language and Education 28 (5), 436-458.

Mazeland, Harrie \& Jan Berenst (2008): Sorting pupils in a report-card meeting: Categorization in a situated activity system. Text \& Talk 28 (1), 55-78.

Michaels, Sarah \& Mary O'Connor (2015): Conceptualizing talk moves as tools: Professional development approaches for academically productive discussions. In Lauren Resnick, Christa Asterhan \& Sherice Clarke (Hrsg.), Socializing intelligence through academic talk and dialogue, 347-361. Washington: American Educational Research Association.

Morek, Miriam (2016): ,Formen mündlicher Darstellung in situ: Zur Komplexität von Diskursanforderungen in Unterrichtsgesprächen. In Olaf Gätje \& Ulrike Behrens (Hrsg.), Mündliches und schriftliches Handeln im Deutschunterricht. Wie Themen entfaltet werden, 95-132. Frankfurt a.M: Peter Lang.

Morek, Miriam, Vivien Heller \& Uta Quasthoff (2017): Argumentieren und Erklären. Konzepte, Modellierungen und empirische Befunde im Rahmen der linguistischen Erwerbs- und Unterrichtsforschung. In Iris Meissner \& Eva Wyss (Hrsg.), Erklären und Argumentieren. Konzepte und Modellierungen in der Angewandten Linguistik, 11-45. Tübingen: Stauffenburg.

Muller Mirza, Nathalie \& Anne-Nelly Perret-Clermont (Hrsg.) (2009): Argumentation and Education. Boston, MA: Springer US.

Prediger, Susanne, Uta Quasthoff, Anna Vogler \& Vivien Heller (2015): How to Elaborate What Teachers Should Learn? Five Steps for Content Specification of Professional Development Programs, Exemplified By “Moves Supporting Participation in Classroom Discussions”. Journal für Mathematik-Didaktik 36 (2), 233-257.

Prediger, Susanne, Kirstin Erath, Uta Quasthoff, Vivien Heller \& Anna Vogler (2016): Befähigung zur Teilhabe an Unterrichtsdiskursen: Die Rolle von Diskurskompetenz. In Jürgen Menthe et al. (Hrsg.), Befähigung zu gesellschaftlicher Teilhabe, 285-300. Münster: Waxmann.

Przyborski, Aglaja (2004): Gesprächsanalyse und dokumentarische Methode. Qualitative Auswertung von Gesprächen, Gruppendiskussionen und anderen Diskursen. Wiesbaden: VS Verlag für Sozialwissenschaften.

Quasthoff, Uta (1978): Bestimmter Artikel und soziale Kategorisierung: Zum Mechanismus von Referenz und Kohärenz. In Dietrich Hartmann, Hansjürgen Linke \& Otto Ludwig (Hrsg.), Sprache in Gegenwart und Geschichte, 154-173. Köln: Böhlau. 
Quasthoff, Uta, Friederike Kern, Sören Ohlhus \& Juliane Stude (2019): Diskurse und Texte von Kindern. Praktiken - Fähigkeiten - Ressourcen: Erwerb. Tübingen: Stauffenburg. http://dx.doi.org/10.17877/DE290R-20360 (Letzter Aufruf am 26. 06.2020).

Quasthoff, Uta \& Susanne Prediger (2017): Fachbezogene Unterrichtsdiskurse zu Beginn der weiterführenden Schule - Interdisziplinäre Untersuchungen zur Unterstützung von sprachlichem und fachlichem Lernen. In Arne Krause, Gesa Lehmann, Winfried Thielmann \& Caroline Trautmann (Hrsg.), Form und Funktion, 625-644. Tübingen: Stauffenburg.

Redder, Angelika (2016): Theoretische Grundlagen der Wissenskonstruktion im Diskurs. In Jörg Kilian, Birgit Brouër \& Dina Lüttenberg (Hrsg.), Handbuch Sprache in der Bildung, 297-318. Berlin, Boston: De Gruyter.

Resnick, Lauren, Christa Asterhan \& Sherice Clarke (2015): Talk, learning, and teaching. In Lauren Resnick, Christa Asterhan \& Sherice Clarke (Hrsg.), Socializing intelligence through academic talk and dialogue, 1-12. Washington: American Educational Research Association.

Reusser, Kurt \& Christine Pauli (2014): Berufsbezogene Überzeugungen von Lehrerinnen und Lehrern. In Ewald Terhart, Hedda Bennewitz \& Martin Rothland (Hrsg.), Handbuch der Forschung zum Lehrerberuf, 642-661. Münster, New York: Waxmann.

Sacks, Harvey (1972): On the Analyzability of Stories by Children. In John Gumperz \& Dell Hymes (Hrsg.), Directions in sociolinguistics. The ethnography of communication, 325-345. Oxford: Blackwell.

Sacks, Harvey (1979): Hotrodder: A revolutionary category. In George Psathas (Hrsg.), Everyday language. Studies in Ethnomethodology, 7-14. New York: Irvington Publishers.

Sacks, Harvey (1989): The M.I.R. Membership Categorization Device. Human Studies 12 (3/4), 271-281.

Sacks, Harvey \& Emanuel Schegloff (1979): Two preferences in the organization of reference to persons in conversation and their interaction. In George Psathas (Hrsg.), Everyday language. Studies in Ethnomethodology, 15-21. New York: Irvington Publishers.

Schlegloff, Emmanuel (1991): Reflections on talk and social structure. In Deirdre Boden \& Don Zimmerman (Hrsg.), Talk and social structure, 44-70. Oxford: Polity.

Seidel, Tina \& Manfred Prenzel (2008): Wie Lehrpersonen Unterricht wahrnehmen und einschätzen - Erfassung pädagogisch-psychologischer Kompetenzen mit Videosequenzen. Zeitschrift für Erziehungswissenschaft, Sonderheft 8. Wiesbaden: VS, 201-216.

Selting, Margret, Peter Auer, Dagmar Barth-Weingarten, Jörg Bergmann, Pia Bergmann, Karin Birkner et al. (2009): Gesprächsanalytisches Transkriptionssystem 2 (GAT 2). Gesprächsforschung - Online-Zeitschrift zur verbalen Interaktion (10), 292-341.

van der Veen, Chiel, Langha de Mey, Claudia van Kruistum \& Bert van Oers (2017): The effect of productive classroom talk and metacommunication on young children's oral communicative competence and subject matter knowledge. An intervention study in early childhood education. Learning and Instruction 48, 14-22.

Vogler, Anna, Susanne Prediger, Uta Quasthoff \& Vivien Heller (2018): Students' and teachers' focus of attention in classroom interaction - subtle sources for the reproduction of social disparities. Mathematics Education Research Journal 30 (3), 299-323. 
Wagner-Willi, Monika (2010). Rituelle Praxis im Spannungsfeld zwischen schulischer Institution und Peer Group. Gruppendiskussionen mit Schülern. In Ralf Bohnsack (Hrsg.), Das Gruppendiskussionsverfahren in der Forschungspraxis, 45-56. Opladen: Budrich.

Yayyusi, Lena (1984): Categorization and the moral order. Boston: Routledge. 
